\title{
General three-point functions in 4D CFT
}

\author{
Emtinan Elkhidir, ${ }^{a}$ Denis Karateev $^{a}$ and Marco Serone ${ }^{a, b}$ \\ ${ }^{a}$ SISSA and INFN, \\ Via Bonomea 265, I-34136 Trieste, Italy \\ ${ }^{b}$ ICTP, \\ Strada Costiera 11, I-34151 Trieste, Italy \\ E-mail: eelkhidi@sissa.it, dkaratee@sissa.it, serone@sissa.it
}

ABSTRACT: We classify and compute, by means of the six-dimensional embedding formalism in twistor space, all possible three-point functions in four dimensional conformal field theories involving bosonic or fermionic operators in irreducible representations of the Lorentz group. We show how to impose in this formalism constraints due to conservation of bosonic or fermionic currents. The number of independent tensor structures appearing in any three-point function is obtained by a simple counting. Using the Operator Product Expansion (OPE), we can then determine the number of structures appearing in 4-point functions with arbitrary operators. This procedure is independent of the way we take the OPE between pairs of operators, namely it is consistent with crossing symmetry, as it should be. An analytic formula for the number of tensor structures for three-point correlators with two symmetric and an arbitrary bosonic (non-conserved) operators is found, which in turn allows to analytically determine the number of structures in 4-point functions of symmetric traceless tensors.

Keywords: Conformal and W Symmetry, Space-Time Symmetries, Higher Spin Symmetry

ARXIV EPRINT: 1412.1796 


\section{Contents}

1 Introduction 1

2 The $6 \mathrm{D}$ embedding formalism in twistor space in an index-free notation 2

3 Three-point functions 5

3.1 Invariant building blocks 6

3.2 Relations between invariants 8

3.3 Final classification of tensor structures and further considerations 9

$\begin{array}{lll}3.4 & 6 \mathrm{D} \text { to } 4 \mathrm{D} \text { dictionary } & 11\end{array}$

$\begin{array}{ll}3.5 & \text { Transformations under 4D parity } \\ \end{array}$

4 Conserved operators $\quad 12$

5 Example: fermion-fermion-tensor correlator $\quad \mathbf{1 4}$

$\begin{array}{lll}5.1 & \text { Non-conserved tensor } & 14\end{array}$

$\begin{array}{lll}5.2 \text { Conserved tensor } & 16\end{array}$

6 Consistency with crossing symmetry: counting four-point function struc$\begin{array}{ll}\text { tures } & 16\end{array}$

$\begin{array}{lll}7 & \text { Conclusions } & 19\end{array}$

$\begin{array}{lr}\text { A Notation and conventions } & 20\end{array}$

B Spinor and vector notation for tensor fields $\quad 22$

\section{Introduction}

Conformal Field Theories (CFTs) play a fundamental role in theoretical physics. For instance, they are the starting and ending points of renormalization group flows in quantum field theories, they describe second-order phase transitions in critical phenomena and, by means of the AdS/CFT correspondence, they can help us in shedding light on various aspects of quantum gravity and string theory. Thanks to the tight constraints imposed by the conformal symmetry, CFTs are also among the few examples (if not the only one) of interacting quantum field theories where exact results are available without supersymmetry in any number of space-time dimensions. In particular, it is well-known that three-point functions of scalar primary operators are univocally determined by the conformal symmetry, up to a coefficient. Three-point functions of arbitrary fields, not only scalars, are also fixed by conformal symmetry up to some coefficients. Most of the attention has been devoted 
to correlators involving traceless symmetric conserved operators,see e.g. refs. [1-8]. More general correlators involving again traceless symmetric (conserved or not) tensors have recently been computed in ref. [9], while some other specific correlator with fermions was considered, e.g., in ref. [10]. Despite these progresses, a general comprehensive computation of three-point functions involving arbitrary fields is not available yet. ${ }^{1}$ The knowledge of such correlators is an important ingredient to extend the recently renewed conformal bootstrap approach [12] beyond scalar correlators and might as well have applications in the AdS/CFT correspondence and in other contexts.

Aim of this paper is to make a step forward along this direction by computing the most general three-point function in four dimensional (4D) CFTs between bosonic or fermionic operators in irreducible representations of the Lorentz group. No extra symmetry (like parity) is assumed. We will achieve this task by extending and generalizing the so called 6D embedding formalism in twistor space as developed by Simmons-Duffin in ref. [13]. As in ref. [13], we will use an index free notation for the correlators, obtained by saturating indices with auxiliary commuting spinors. Constraints coming from bosonic or fermionic conserved currents can be simply worked out in this formalism. We will see, generalizing the results found in ref. [9] for traceless symmetric operators, that $4 \mathrm{D}$ current conservation conditions can be covariantly lifted to $6 \mathrm{D}$ only if the conserved operator saturates the unitarity bound.

The extension of our formalism to higher-point functions is in principle straightforward, but technically complicated. Using the OPE, however, we can at least determine the number of structures appearing in higher-point functions with arbitrary operators by using our result for three-point functions. We have in particular computed in closed form the number of structures appearing in certain 4-point functions with traceless symmetric operators and checked the consistency of the result using crossing symmetry.

The structure of the paper is as follows. In section 2 we will review the $6 \mathrm{D}$ embedding formalism in twistor space in index-free notation and set-up our notation. We classify and compute all possible three-point functions in section 3. This is the key section of the paper, with eq. (3.31) being the most important result of this work. We show in section 4 how the additional constraints imposed by conserved currents are implemented in the $6 \mathrm{D}$ twistor space. The key relations of this section are eqs. (4.10) and (4.11). In order to show the power and simplicity of our formalism, in section 5 we work out explicitly some examples of correlators, with and without conserved operators. In section 6 we show how our results can be used to compute the number of independent tensor structures of four-point functions and their consistency with crossing symmetry. We conclude in section 7. Our notation and conventions, as well as useful relations, are summarized in appendix A, while in appendix $\mathrm{B}$ we recall the map between the vector and spinor notation for tensor fields.

\section{The $6 \mathrm{D}$ embedding formalism in twistor space in an index-free notation}

The embedding formalism idea dates back to Dirac [15]. It is based on the simple observation that the $4 \mathrm{D}$ conformal group is isomorphic to $\mathrm{SO}(4,2)$, that is the Lorentz group of a $6 \mathrm{D}$ flat space with signature $(--++++)$. The non-linear action of the conformal group

\footnotetext{
${ }^{1}$ See ref. [11] for an early attempt.
} 
in $4 \mathrm{D}$ turns into simple linear Lorentz transformations in 6D. Hence, by properly extending $4 \mathrm{D}$ fields to $6 \mathrm{D}$, one can more easily derive the constraints imposed by the conformal symmetry on the correlation functions. The embedding formalism has successfully been used in ordinary space to study correlation functions of traceless symmetric tensors $[9,10,16-$ 18] (see also ref. [19]). Using the local isomorphism between $\mathrm{SO}(4,2)$ and $\mathrm{SU}(2,2)$, the embedding formalism can be reformulated in twistor space. In this form it has sporadically been used in the literature, mainly in the context of super conformal field theories (see e.g. refs. [20-25]). More recently, it has been applied in ref. [13] to study correlation functions in 4D CFTs. For completeness, we briefly review here the embedding formalism in twistor space, essentially following the analysis made in section 5 of ref. [13]. We assume that the reader is familiar with basics of CFT.

On $R^{4,2}$, we consider the light-cone defined by (see appendix $\mathrm{A}$ for our notations and conventions)

$$
X^{2}=X^{M} X^{N} \eta_{M N}=\eta_{\mu \nu} X^{\mu} X^{\nu}+X^{+} X^{-}=0 .
$$

We define a projective light-cone by identifying (on the cone) $X^{M} \cong \lambda X^{M}$, with $\lambda$ any real non-vanishing constant. In this way, we have a map between $6 \mathrm{D}$ and $4 \mathrm{D}$ coordinates. The standard 4D coordinates $x^{\mu}$ should not depend on $\lambda$ and are defined as

$$
x^{\mu}=\frac{X^{\mu}}{X^{+}} \text {. }
$$

It can be shown that conformal transformations acting on $x^{\mu}$ are mapped to Lorentz transformations acting on the light-cone.

Let us now consider how 4D fields are uplifted to $6 \mathrm{D}$, starting with scalar fields. Let $\phi(x)$ be a $4 \mathrm{D}$ primary scalar operator with scaling dimension $\Delta$ and $\Phi(X)$ its corresponding $6 \mathrm{D}$ field. In order to be well defined on the projective cone, $\Phi(X)$ should be a homogeneous function: $\Phi(\lambda X)=\lambda^{-n} \Phi(X)$, for some $n$. A natural identification is

$$
\phi(x)=\left(X^{+}\right)^{n} \Phi(X) .
$$

It is easy to verify that $n=\Delta$ in eq. (2.3) to correctly reproduce the conformal transformations of $\phi(x)$. Let us now consider spin $1 / 2$ primary fermions $\psi_{\alpha}(x)$ and $\bar{\phi}^{\dot{\alpha}}(x)$, with scaling dimension $\Delta$. As shown in ref. [10], such fields are uplifted to 6D homogeneous twistors $\Psi_{a}(X)$ and $\bar{\Phi}^{a}(X)$, with degree $n=\Delta-1 / 2$. A transversality condition is imposed on the $6 \mathrm{D}$ fields, in order to match the number of degrees of freedom:

$$
\begin{aligned}
& \overline{\mathbf{X}}^{a b} \Psi_{b}(X)=0, \\
& \bar{\Phi}^{a}(X) \mathbf{X}_{a b}=0,
\end{aligned}
$$

where $\mathbf{X}$ and $\overline{\mathbf{X}}$ are twistor space-time coordinates, defined in eq. (A.11). By solving eq. (2.4), we get

$$
\begin{aligned}
& \Psi_{a}(X)=\left(X^{+}\right)^{-\Delta+1 / 2}\left(\begin{array}{c}
\psi_{\alpha}(x) \\
-\left(x_{\mu} \bar{\sigma}^{\mu}\right)^{\dot{\alpha} \beta} \psi_{\beta}(x)
\end{array}\right), \\
& \bar{\Phi}^{a}(X)=\left(X^{+}\right)^{-\Delta+1 / 2}\left(\begin{array}{c}
\bar{\phi}_{\dot{\beta}}(x)\left(x_{\mu} \bar{\sigma}^{\mu}\right)^{\dot{\beta} \alpha} \\
\bar{\phi}_{\dot{\alpha}}(x)
\end{array}\right) .
\end{aligned}
$$


As discussed in ref. [13], it is more convenient to embed $\psi_{\alpha}(x)$ and $\bar{\phi}^{\dot{\alpha}}(x)$ to twistors $\bar{\Psi}^{a}(X)$ and $\Phi_{a}(X)$, respectively, with degree $n=\Delta+1 / 2$. In this way, we essentially trade the transversality condition for a gauge redundancy. A generic solution of eq. (2.4) is given by $\Psi=\mathbf{X} \bar{\Psi}$ and $\bar{\Phi}=\Phi \overline{\mathbf{X}}$ for some $\bar{\Psi}$ and $\Phi$, since on the cone

$$
\mathbf{X} \overline{\mathbf{X}}=\overline{\mathbf{X}} \mathbf{X}=0 .
$$

We can then equivalently associate $\psi_{\alpha}(x)$ to a twistor $\bar{\Psi}^{a}(X)$, and $\bar{\phi}^{\dot{\alpha}}(x)$ to a twistor $\Phi_{a}(X)$ as follows:

$$
\begin{aligned}
\psi_{\alpha}(x) & =\left(X^{+}\right)^{\Delta-1 / 2} \mathbf{X}_{\alpha a} \bar{\Psi}^{a}(X), \\
\bar{\phi}^{\dot{\alpha}}(x) & =\left(X^{+}\right)^{\Delta-1 / 2} \overline{\mathbf{X}}^{\dot{\alpha} a} \Phi_{a}(X),
\end{aligned}
$$

where $\overline{\mathbf{X}}^{\dot{\beta} b}=\epsilon^{\dot{\beta} \dot{\gamma}} \overline{\mathbf{X}}_{\dot{\gamma}}^{b}$. The twistors $\bar{\Psi}(X)$ and $\Phi(X)$ are subject to an equivalence relation,

$$
\begin{aligned}
& \bar{\Psi}(X) \sim \bar{\Psi}(X)+\overline{\mathbf{X}} V, \\
& \Phi(X) \sim \Phi(X)+\mathbf{X} \bar{W},
\end{aligned}
$$

with $V$ and $\bar{W}$ generic twistors. We are now ready to consider a 4 D primary spinor-tensor in an arbitrary irreducible representation of the Lorentz group, with scaling dimension $\Delta$ :

$$
f_{\alpha_{1} \ldots \alpha_{l}}^{\dot{\beta}_{1} \ldots \dot{\beta}_{\bar{l}}}(x),
$$

where dotted and undotted indices are symmetrized. We will denote such a representation as $(l, \bar{l})$, namely by the number of undotted and dotted indices that appear. Hence, a spin $1 / 2$ Weyl fermion will be in the $(1,0)$ or $(0,1)$, a vector in the $(1,1)$, an antisymmetric tensor in the $(2,0) \oplus(0,2)$ and so on. Generalizing eq. (2.7), we encode $f_{\alpha_{1} \ldots \alpha_{l}}^{\dot{\beta}_{1} \ldots \dot{\beta}_{\bar{l}}}$ in a $6 \mathrm{D}$ multi-twistor field $F_{b_{1} \ldots b_{\bar{l}}}^{a_{1} \ldots a_{l}}$ of degree $n=\Delta+(l+\bar{l}) / 2$ as follows:

$$
f_{\alpha_{1} \ldots \alpha_{l}}^{\dot{\beta}_{1} \ldots \dot{\beta}_{\bar{l}}}(x)=\left(X^{+}\right)^{\Delta-(l+\bar{l}) / 2} \mathbf{X}_{\alpha_{1} a_{1}} \ldots \mathbf{X}_{\alpha_{l} a_{l}} \overline{\mathbf{X}}^{\dot{\beta}_{1} b_{1}} \ldots \overline{\mathbf{X}}^{\dot{\beta}_{\bar{l}} b_{\bar{l}}} F_{b_{1} \ldots b_{\bar{l}}}^{a_{1} \ldots a_{l}}(X) .
$$

Given the gauge redundancy (2.8) in each index, the $4 \mathrm{D}$ field $f$ is uplifted to an equivalence class of 6 D fields $F$. Any two fields $F$ and $\hat{F}=F+\overline{\mathbf{X}} V$ or $\hat{F}=F+\mathbf{X} \bar{W}$, for some multi twistors $V$ and $\bar{W}$, are equivalent uplifts of $f$, because of eq. (2.6). There is yet another equivalence class, due again to eq. (2.6). Twistors of the form $F_{b_{1} b_{2} \ldots}^{a_{1} \ldots}=\delta_{b_{1}}^{a_{1}} Z_{b_{2} \ldots}^{a_{2} \ldots}$ give a vanishing contribution in eq. (2.10). Hence, without loss of generality, we can take as uplift of $f$ a multi-twistor $F$ with vanishing trace, namely:

$$
\delta_{a_{i}}^{b_{j}} F_{b_{1} \ldots b_{\bar{l}}}^{a_{1} \ldots a_{l}}(X)=0, \quad \forall i=1, \ldots, l, \forall j=1, \ldots, \bar{l} .
$$

It is very useful to use an index-free notation by defining

$$
f(x, s, \bar{s}) \equiv f_{\alpha_{1} \ldots \alpha_{l}}^{\dot{\beta}_{1} \ldots \dot{\beta}_{\bar{l}}}(x) s^{\alpha_{1}} \ldots s^{\alpha_{l}} \bar{s}_{\dot{\beta}_{1}} \ldots \bar{s}_{\dot{\beta}_{\bar{l}}},
$$

where $s^{\alpha}$ and $\bar{s}_{\dot{\beta}}$ are auxiliary (commuting and independent) spinors. Similarly, we define

$$
F(X, S, \bar{S}) \equiv\left(X^{+}\right)^{\Delta+(l+\bar{l}) / 2} F_{b_{1} \ldots b_{\bar{l}}}^{a_{1} \ldots a_{l}}(X) S_{a_{1}} \ldots S_{a_{l}} \bar{S}^{b_{1}} \ldots \bar{S}^{b_{\bar{l}}}
$$


in terms of auxiliary (again commuting and independent) twistors $S_{a}$ and $\bar{S}^{a}$. Consistency of eqs. (2.10), (2.12) and (2.13) implies that

$$
S_{a}=s^{\alpha} \frac{\mathbf{X}_{\alpha a}}{X^{+}}, \quad \bar{S}^{a}=\bar{s}_{\dot{\beta}} \frac{\overline{\mathbf{X}}^{\dot{\beta} a}}{X^{+}} .
$$

From eq. (2.14) we also deduce

$$
\overline{\mathbf{X}}^{a b} S_{b}=\bar{S}^{b} \mathbf{X}_{b a}=\bar{S}^{a} S_{a}=0
$$

consistently with the gauge redundancies we have in choosing $F$. Given a $6 \mathrm{D}$ multi-twistor field $F$, the corresponding $4 \mathrm{D}$ field $f$ is explicitly given by

$$
f_{\alpha_{1} \ldots \alpha_{l}}^{\dot{\beta}_{1} \ldots \dot{\beta}_{\bar{l}}}(x)=\frac{1}{l ! \bar{l} !} \frac{\partial}{\partial s^{\alpha_{1}}} \ldots \frac{\partial}{\partial s^{\alpha_{l}}} \frac{\partial}{\partial \bar{s}_{\dot{\beta}_{1}}} \ldots \frac{\partial}{\partial \bar{s}_{\dot{\beta}_{\bar{l}}}} F\left(X, \frac{s \mathbf{X}}{X^{+}}, \frac{\bar{s} \overline{\mathbf{X}}}{X^{+}}\right) .
$$

It is useful to compare the index-free notation introduced here with the one introduced in ref. [9] for symmetric traceless tensors in terms of polynomials in auxiliary variables $z^{\mu}$ and $Z^{M}$. Recall that in vector notation, a $4 \mathrm{D}$ symmetric traceless tensor $t_{\mu_{1} \ldots \mu_{l}}$ can be embedded in a $6 \mathrm{D}$ tensor $T_{M_{1} \ldots M_{l}}$ by means of the relation

$$
t_{\mu_{1} \ldots \mu_{l}}=\left(X^{+}\right)^{\Delta-l} \frac{\partial X^{M_{1}}}{\partial x^{\mu_{1}}} \ldots \frac{\partial X^{M_{l}}}{\partial x^{\mu_{l}}} T_{M_{1} \ldots M_{l}}
$$

where $T_{M_{1} \ldots M_{l}}$ is symmetric traceless in $6 \mathrm{D}$, homogeneous of degree $\Delta$, as well as transverse: $X^{A_{1}} T_{A_{1} A_{2} \ldots A_{l}}=0$. In ref. [9], $4 \mathrm{D}$ and $6 \mathrm{D}$ fields are encoded in the polynomials

$$
\begin{aligned}
t(x, z) & =t_{\mu_{1} \ldots \mu_{n}} z^{\mu_{1}} \ldots z^{\mu_{n}} \\
T(X, Z) & =\left(X^{+}\right)^{\Delta} T_{M_{1} \ldots M_{n}} Z^{M_{1}} \ldots Z^{M_{n}},
\end{aligned}
$$

where in Minkowski space $z_{\mu}$ is a light-cone vector, $z_{\mu} z^{\mu}=0$. A null vector can always be written as a product of two spinors:

$$
z^{\mu}=\sigma_{\alpha \dot{\beta}}^{\mu} s^{\alpha} \bar{s}^{\dot{\beta}}
$$

Given the relation (B.6) between symmetric traceless tensors written in vector and spinor notation, the spinors $s^{\alpha}$ and $\bar{s}^{\dot{\alpha}}$ appearing in eq. (2.19) are exactly the ones defined in eq. (2.12). On the contrary, there is not a simple relation between the $6 \mathrm{D}$ coordinates $Z^{A}$ and the $6 \mathrm{D}$ twistors $S_{a}$ and $\bar{S}^{a}$.

\section{Three-point functions}

The goal of this section is to classify and compute the most general three-point function in a 4D CFT using the 6D embedding formalism reviewed in section 2, essentially completing the program that was outlined in ref. [13], where this formalism was first proposed and used. Although some of the results of this section were already obtained in ref. [13], for the clarity of the presentation and for completeness, they will be reported here in a more systematic framework. 
Three-point functions in a CFT are completely fixed by the conformal symmetry, up to a set of constants. Let us denote by $F_{i}=F_{i}\left(X_{i}, S_{i}, \bar{S}_{i}\right)$ the index-free $6 \mathrm{D}$ multi tensor field corresponding to some $\left(l_{i}, \bar{l}_{i}\right) 4 \mathrm{D}$ tensor field $f_{i}$. An arbitrary three-point function can schematically be written as

$$
\left\langle F_{1} F_{2} F_{3}\right\rangle=\mathcal{K} \sum_{s=1}^{N_{3}} \lambda_{s} \mathcal{T}_{s}
$$

where $\mathcal{K}$ is a kinematic factor which depend on the scaling dimension and spin of the external fields, $\mathcal{T}_{s}$ are dimensionless (i.e. homogeneous with degree zero) $\mathrm{SU}(2,2)$ invariant tensor structures which encode the Lorentz structure of the fields and $\lambda_{s}$ are constants. The index $s$ runs over all the possible different independent tensor structures compatible with conformal invariance.

Let us start with the kinematic factor

$$
\mathcal{K}=\frac{1}{X_{12}^{a_{12}} X_{13}^{a_{13}} X_{23}^{a_{23}}}
$$

where we use a $6 \mathrm{D}$ short-hand notation

$$
X_{i j} \equiv X_{i} \cdot X_{j}
$$

The coefficients $a_{i j}$ are determined by matching the scaling dimension of both sides of eq. (3.1):

$$
a_{i j}=\frac{1}{2}\left(\Delta_{i j k}+\frac{\left(l_{i}+\bar{l}_{i}\right)+\left(l_{j}+\bar{l}_{j}\right)-\left(l_{k}+\bar{l}_{k}\right)}{2}\right), \quad i \neq j \neq k,
$$

where we have defined

$$
\Delta_{i j k} \equiv \Delta_{i}+\Delta_{j}-\Delta_{k}=\Delta_{j i k} .
$$

Finding the tensor structures $\mathcal{T}_{s}$ is a much less trivial problem. Any $\mathcal{T}_{s}$ will be a product of some fundamental $\mathrm{SU}(2,2)$ invariant building blocks that are to be determined.

\subsection{Invariant building blocks}

The fundamental group-theoretical objects carrying $\mathrm{SU}(2,2)$ indices, which should eventually be combined with the auxiliary twistors $S_{a}$ and $\bar{S}^{b}$ to form $\mathrm{SU}(2,2)$ invariants, are obtained as products of

$$
\delta_{b}^{a}, \varepsilon_{a b c d}, \varepsilon^{a b c d}, \mathbf{X}_{a b}, \overline{\mathbf{X}}^{a b}
$$

Let us first focus on the $\varepsilon$ tensors. Their contraction with any other object in eq. (3.6) does not give any new structures, because they reduce to a sum of already existing elements in eq. (3.6), for example:

$$
\begin{aligned}
\varepsilon^{a b c d} \varepsilon_{a e f g} & =\delta_{e}^{b} \delta_{f}^{c} \delta_{g}^{d}-\delta_{e}^{b} \delta_{g}^{c} \delta_{f}^{d}-\delta_{f}^{b} \delta_{e}^{c} \delta_{g}^{d}+\delta_{f}^{b} \delta_{g}^{c} \delta_{e}^{d}+\delta_{g}^{b} \delta_{e}^{c} \delta_{f}^{d}-\delta_{g}^{b} \delta_{f}^{c} \delta_{e}^{d} \\
\varepsilon^{a b c d} \mathbf{X}_{a e} & =-\delta_{e}^{b} \overline{\mathbf{X}}^{c d}+\delta_{e}^{c} \overline{\mathbf{X}}^{b d}-\delta_{e}^{d} \overline{\mathbf{X}}^{b c}
\end{aligned}
$$

Actually, for three-point functions the $\varepsilon$-symbols drop from the discussion completely. It can be seen using the index-free formalism where $\varepsilon$ is encoded into $\varepsilon_{a b c d} \bar{S}_{i}^{a} \bar{S}_{j}^{b} \bar{S}_{k}^{c} \bar{S}_{l}^{d}$, which 
vanishes unless $i \neq j \neq k \neq l$. Thus, the tensors $\varepsilon$ become relevant starting from the four-point functions. The fundamental group-theoretical objects can be grouped into three sets

$$
\left\{\delta_{a}^{b},\left[\mathbf{X}_{i} \overline{\mathbf{X}}_{j}\right]_{a}^{b},\left[\mathbf{X}_{i} \overline{\mathbf{X}}_{j} \mathbf{X}_{k} \overline{\mathbf{X}}_{l}\right]_{a}^{b}, \ldots\right\},\left\{\left[\overline{\mathbf{X}}_{i}\right]^{a b},\left[\overline{\mathbf{X}}_{i} \mathbf{X}_{j} \overline{\mathbf{X}}_{k}\right]^{a b}, \ldots\right\},\left\{\left[\mathbf{X}_{i}\right]_{a b},\left[\mathbf{X}_{i} \overline{\mathbf{X}}_{j} \mathbf{X}_{k}\right]_{a b}, \ldots\right\}
$$

Multiplying these objects by auxiliary twistors $S$ and $\bar{S}$ will give us the $\mathrm{SU}(2,2)$ invariant building blocks needed to characterize the three-point (or any other $n$-point) function. They are not all independent, given the relations (2.6), (2.15) and (A.13).

Let us first determine the general form of two-point functions $\left\langle F_{1} F_{2}\right\rangle$. It is clear in this case that the only non-vanishing independent $\mathrm{SU}(2,2)$ invariant is obtained by contracting one twistor $\bar{S}_{1}$ with $S_{2}$ or viceversa. The form of the two-point function is uniquely determined:

$$
\left\langle F_{1}\left(X_{1}, S_{1}, \bar{S}_{1}\right) F_{2}\left(X_{2}, S_{2}, \bar{S}_{2}\right)\right\rangle=c X_{12}^{2 \Delta_{1}} I_{21}^{l_{1}} \bar{I}_{12}^{\bar{l}_{1}} \delta_{l_{1}, \bar{l}_{2}} \delta_{l_{2}, \bar{l}_{1}} \delta_{\Delta_{1}, \Delta_{2}}
$$

where $c$ is a normalization factor and we have defined the $\mathrm{SU}(2,2)$ invariant

$$
I_{i j} \equiv \bar{S}_{i} S_{j}
$$

For three-point functions three more invariants arise:

$$
\begin{aligned}
K_{i, j k} & \equiv N_{i, j k} S_{j} \overline{\mathbf{X}}_{i} S_{k}, \\
\bar{K}_{i, j k} & \equiv N_{i, j k} \bar{S}_{j} \mathbf{X}_{i} \bar{S}_{k}, \\
J_{i, j k} & \equiv N_{j k} \bar{S}_{i} \mathbf{X}_{j} \overline{\mathbf{X}}_{k} S_{i}
\end{aligned}
$$

The normalization factors

$$
N_{j k} \equiv \frac{1}{X_{j k}}, \quad N_{i, j k} \equiv \sqrt{\frac{X_{j k}}{X_{i j} X_{i k}}},
$$

are introduced to make the $\mathrm{SU}(2,2)$ invariants in eqs. (3.11)-(3.14) dimensionless and welldefined on the $6 \mathrm{D}$ light-cone. ${ }^{2}$ Notice that in eqs. (3.12)-(3.14) $i \neq j \neq k$ and indices are not summed. The invariants (3.12)-(3.14) are all anti-symmetric in the two indices after the comma:

$$
K_{i, j k}=-K_{i, k j}, \quad \bar{K}_{i, j k}=-\bar{K}_{i, k j}, \quad J_{i, j k}=-J_{i, k j},
$$

due to the anti-symmetry of $\mathbf{X}, \overline{\mathbf{X}}$ and the relations (2.15), (A.13).

Every other $\mathrm{SU}(2,2)$ invariant object obtained from eq. (3.9) can be written in terms of different combinations of $I_{i j}, K_{i, j k}, \bar{K}_{i, j k}$ and $J_{i, j k}$. Using eqs. (3.11)-(3.14), the most general tensor structure can be written as follows:

$$
\mathcal{T}_{s}=\lambda_{s} I_{12}^{m_{12}} I_{21}^{m_{21}} I_{13}^{m_{13}} I_{31}^{m_{31}} I_{23}^{m_{23}} I_{32}^{m_{32}} K_{1,23}^{k_{1}} K_{2,13}^{k_{2}} K_{3,12}^{k_{3}} \bar{K}_{1,23}^{\bar{k}_{1}} \bar{K}_{2,13}^{\bar{k}_{2}} \bar{K}_{3,12}^{\bar{k}_{3}} J_{1,23}^{j_{1}} J_{2,13}^{j_{2}} J_{3,12}^{j_{3}}
$$

\footnotetext{
${ }^{2}$ Notice the different normalization and slight different index notation in the definition of the invariants $I, K, \bar{K}$ and $J$ with respect to the ones defined in ref. [13].
} 
where $m_{i j}, k_{i}, \bar{k}_{i}$ and $j_{i}$ are a set of non-negative integers. Matching the powers of $S_{i}$ and $\bar{S}_{j}$ in both sides of eq. (3.1) gives us six constraints:

$$
\left\{\begin{array}{l}
l_{1}=m_{21}+m_{31}+0+k_{2}+k_{3}+j_{1} \\
l_{2}=m_{12}+m_{32}+k_{1}+0+k_{3}+j_{2} \\
l_{3}=m_{13}+m_{23}+k_{1}+k_{2}+0+j_{3} \\
\bar{l}_{1}=m_{12}+m_{13}+0+\bar{k}_{2}+\bar{k}_{3}+j_{1} \\
\bar{l}_{2}=m_{21}+m_{23}+\bar{k}_{1}+0+\bar{k}_{3}+j_{2} \\
\bar{l}_{3}=m_{31}+m_{32}+\bar{k}_{1}+\bar{k}_{2}+0+j_{3} .
\end{array}\right.
$$

This would have completed the classification of the three-point functions if the tensor structures $\mathcal{T}_{s}$ were all linearly independent, but they are not, and hence a more refined analysis is necessary.

\subsection{Relations between invariants}

The dependence of the structures (3.17) has its roots in a set of identities among the twistors $S_{i}$ and the coordinates $\mathbf{X}_{j}$, when $i=j$. Recall that on the $6 \mathrm{D}$ light-cone $\mathbf{X}$ can be written in terms of auxiliary twistors $V$ and $W$ :

$$
\mathbf{X}_{a b}=V_{a} W_{b}-V_{b} W_{a} .
$$

The twistor $S$ can also be rewritten in an analogous manner. We solve eq. (2.15) by $S_{a}=\mathbf{X}_{a b} \bar{T}^{b}$ for some $\bar{T}^{b}$ and then we use eq. (3.19) to get

$$
S_{a}=\alpha V_{a}+\beta W_{a}
$$

with $\alpha=\bar{T} W, \beta=-\bar{T} V$. Using eqs. (3.19) and (3.20) it is immediate to verify the identities

$$
\begin{aligned}
S_{a} \mathbf{X}_{b c}+S_{b} \mathbf{X}_{c a}+S_{c} \mathbf{X}_{a b} & =0 \\
\mathbf{X}_{a b} \mathbf{X}_{c d}+\mathbf{X}_{c a} \mathbf{X}_{b d}+\mathbf{X}_{b c} \mathbf{X}_{a d} & =0 .
\end{aligned}
$$

Analogous relations apply for the dual twistors $\bar{S}$ and $\overline{\mathbf{X}}$. We have not found identities involving more $S$ 's or X's that do not boil down to eqs. (3.21) and (3.22). Applying eqs. (3.21) and (3.22) (actually it is enough to use only eq. (3.21)) to bi-products of invariants we get the following relations (no sum over indices):

$$
\begin{aligned}
K_{j, i k} \bar{K}_{i, j k} & =2 I_{k i} I_{j k}-I_{j i} J_{k, i j}, \\
J_{i, j k} J_{j, i k} & =2\left(2 I_{i j} I_{j i}+K_{k, i j} \bar{K}_{k, i j}\right), \\
J_{j, i k} K_{j, i k} & =2\left(-I_{j i} K_{i, j k}+I_{j k} K_{k, i j}\right), \\
J_{j, i k} \bar{K}_{j, i k} & =2\left(-I_{i j} \bar{K}_{i, k j}-I_{k j} \bar{K}_{k, i j}\right) .
\end{aligned}
$$

We have verified that higher order relations involving more than 2 invariants always arise as the composition of the relations (3.23)-(3.26). This is expected, since the fundamental 
identities (3.21) and (3.22) involve only two tensors. A particularly useful third-order relation is

$$
J_{1,23} J_{2,13} J_{3,12}=8\left(I_{21} I_{13} I_{32}-I_{12} I_{31} I_{23}\right)+4\left(I_{23} I_{32} J_{1,23}-I_{13} I_{31} J_{2,13}+I_{12} I_{21} J_{3,12}\right),
$$

which is obtained by applying, in order, eqs. (3.24), (3.26) and (3.23). The relations (3.23)(3.27) have been originally obtained in ref. [13], though it was not clear there whether additional relations were possible.

Combining eqs. (3.23) and (3.24), we see that a product of any $K$ and $\bar{K}$ can be reduced to a combination of $I$ 's and $J$ 's. Thus, we obtain the first constraint on the integers $k_{i}$ and $\bar{k}_{i}$ appearing in eq. (3.17):

$$
k_{1}=k_{2}=k_{3}=0 \quad \text { or } \quad \bar{k}_{1}=\bar{k}_{2}=\bar{k}_{3}=0 .
$$

In other words, we can always choose a basis of invariants $\mathcal{T}_{s}$ where $K$ 's and $\bar{K}$ 's never appear together. Next we can apply eq. (3.27) successively. At each step the tensor structure splits into five ones, each time with a reduced number of $J$ 's. We keep applying eq. (3.27) until the initial tensor structure is written as a sum of tensor structures where all have at least one value of $j_{1}, j_{2}$, or $j_{3}$ equal to zero. Thus we get the second constraint in eq. (3.17):

$$
j_{1}=0 \quad \text { or } \quad j_{2}=0 \text { or } j_{3}=0 .
$$

The last step is to apply eq. (3.26) (for $k_{1,2,3}=0$ ) or eq. (3.25) (for $\bar{k}_{1,2,3}=0$ ), so that products of the form $K_{i, \ldots} J_{i, \ldots}$ or $\bar{K}_{i, \ldots} J_{i, \ldots}$ can be rewritten using only $K$ 's or $\bar{K}$ 's of a different type. It is not difficult to convince oneself that this boils down to the following further constraints on eq. (3.17):

$$
\left\{\begin{array} { l l l } 
{ k _ { 1 } = 0 } & { \text { or } } & { j _ { 1 } = 0 } \\
{ k _ { 2 } = 0 } & { \text { or } } & { j _ { 2 } = 0 } \\
{ k _ { 3 } = 0 } & { \text { or } } & { j _ { 3 } = 0 }
\end{array} \quad \left\{\begin{array}{ccc}
\bar{k}_{1}=0 & \text { or } & j_{1}=0 \\
\bar{k}_{2}=0 & \text { or } & j_{2}=0 \\
\bar{k}_{3}=0 & \text { or } & j_{3}=0
\end{array}\right.\right.
$$

\subsection{Final classification of tensor structures and further considerations}

There are no further relations to be imposed so we can finally state the main result of this paper.

The most general three-point function $\left\langle F_{1} F_{2} F_{3}\right\rangle$ can be written as

$$
\left\langle F_{1} F_{2} F_{3}\right\rangle=\mathcal{K} \sum_{s=1}^{N_{3}} \lambda_{s}\left(\prod_{i \neq j=1}^{3} I_{i j}^{m_{i j}}\right) K_{1,23}^{k_{1}} K_{2,13}^{k_{2}} K_{3,12}^{k_{3}} \bar{K}_{1,23} \bar{k}_{1} \bar{K}_{2,13} \bar{k}_{2} \bar{K}_{3,12} \bar{k}_{3} J_{1,23}^{j_{1}} J_{2,13}^{j_{2}} J_{3,12}^{j_{3}},
$$

where $\mathcal{K}$ is given by eq. (3.2) and $s$ runs over all the independent tensor structures. These are given by the set of non-negative exponents $m_{i j}, k_{i}, \bar{k}_{i}$ and $j_{i}$ solution of eq. (3.18) and subjected to the constraints (3.28), (3.29) and (3.30). The latter require that, modulo the $I_{i j}$ invariants, at most three more invariants can be present in each tensor structure. We can have i) $2 J$ 's, ii) $3 K$ 's, iii) $2 K$ 's and $1 J$, iv) $1 K$ and $2 J$ 's, v) $3 \bar{K}$ 's, vi) $2 \bar{K}$ 's and 1 $J$, vii) $1 \bar{K}$ and $2 J$ 's. 
Let us discuss some implications of eq. (3.31). It is useful to define

$$
\Delta l \equiv l_{1}+l_{2}+l_{3}-\left(\bar{l}_{1}+\bar{l}_{2}+\bar{l}_{3}\right) .
$$

Using the system (3.18), we immediately get

$$
\begin{aligned}
\Delta l & =2\left(k_{1}+k_{2}+k_{3}-\bar{k}_{1}-\bar{k}_{2}-\bar{k}_{3}\right), \\
k_{1}+k_{2}+k_{3} & \leq \min \left(l_{1}+l_{2}, l_{1}+l_{3}, l_{2}+l_{3}\right), \quad \bar{k}_{1}+\bar{k}_{2}+\bar{k}_{3} \leq \min \left(\bar{l}_{1}+\bar{l}_{2}, \bar{l}_{1}+\bar{l}_{3}, \bar{l}_{2}+\bar{l}_{3}\right),
\end{aligned}
$$

and hence

$$
-2 \min \left(\bar{l}_{1}+\bar{l}_{2}, \bar{l}_{1}+\bar{l}_{3}, \bar{l}_{2}+\bar{l}_{3}\right) \leq \Delta l \leq 2 \min \left(l_{1}+l_{2}, l_{1}+l_{3}, l_{2}+l_{3}\right) .
$$

These are the conditions for the $4 \mathrm{D}$ three-point function $\left\langle f_{1} f_{2} f_{3}\right\rangle$ to be non-vanishing. They exactly match the findings of ref. [26]. Indeed, in that paper it was found that the 3 -point function $\left\langle f_{1} f_{2} f_{3}\right\rangle$, with $f_{i}$ primary fields in the $\left(l_{i}, \bar{l}_{i}\right)$ representations of $\mathrm{SL}(2, C)$, is non-vanishing if the decomposition of the tensor product $\left(l_{1}, \bar{l}_{1}\right) \otimes\left(l_{2}, \bar{l}_{2}\right) \otimes\left(l_{3}, \bar{l}_{3}\right)$ contains a traceless-symmetric representation $(l, l)$. Then we have

$\left(l_{1}, \bar{l}_{1}\right) \otimes\left(l_{2}, \bar{l}_{2}\right) \otimes\left(l_{3}, \bar{l}_{3}\right)=\sum_{m=0}^{\min \left(l_{1}, l_{2}\right)} \sum_{\bar{m}=0}^{\min \left(\bar{l}_{1}, \bar{l}_{2}\right)} \sum_{p=0}^{p_{m}} \sum_{\bar{p}=0}^{\bar{p}_{m}}\left(l_{1}+l_{2}+l_{3}-2 m-2 p, \bar{l}_{1}+\bar{l}_{2}+\bar{l}_{3}-2 \bar{m}-2 \bar{p}\right)$,

where $p_{m}=\min \left(l_{1}+l_{2}-2 m, l_{3}\right), \bar{p}_{m}=\min \left(\bar{l}_{1}+\bar{l}_{2}-2 \bar{m}, \bar{l}_{3}\right)$, and the indices of summation are subjected to the following constraints

$$
m+p \leq \min \left(l_{1}+l_{2}, l_{1}+l_{3}, l_{2}+l_{3}\right), \quad \bar{m}+\bar{p} \leq \min \left(\bar{l}_{1}+\bar{l}_{2}, \bar{l}_{1}+\bar{l}_{3}, \bar{l}_{2}+\bar{l}_{3}\right) .
$$

Demanding that a term of the form $(l, l)$ appears in the r.h.s. of eq. (3.35) implies

$$
\Delta l=2(m+p-\bar{m}-\bar{p}),
$$

where $\Delta l$ is defined in eq. (3.32). We then see that eqs. (3.36) and (3.37) exactly correspond to eqs. (3.33), with the identification $m+p \rightarrow k_{1}+k_{2}+k_{3}, \bar{m}+\bar{p} \rightarrow \bar{k}_{1}+\bar{k}_{2}+\bar{k}_{3}$.

The master formula (3.31) computes the most general three-point function compatible with conformal symmetry. Invariance under parity transformations, in particular, is not assumed. It should be obvious that additional symmetries (like exchange symmetries with identical operators or conserved operators) put further constrains on the form of the 3-point function. We will consider in more detail parity in subsection 3.5 and conserved operators in section 4 .

For any given correlator, the explicit form and the number $N_{3}$ of independent tensor structures is easily determined with a numerical algorithm. In general, $N_{3}=$ $N_{3}\left(l_{1}, \bar{l}_{1}, l_{2}, \bar{l}_{2}, l_{3}, \bar{l}_{3}\right)$ and it is a laborius task (which we have not tried to do) to find its analytic expression for any correlator. However, we have been able to get a simple formula for the correlations involving two traceless-symmetric tensors $\left(l_{1}, l_{1}\right),\left(l_{2}, l_{2}\right)$ and an arbitrary $\left(l_{3}, \bar{l}_{3}\right)$ field. The number of independent structures is found to be

$$
\begin{aligned}
N_{3}\left(l_{1}, l_{1}, l_{2}, l_{2}, l_{3}, \bar{l}_{3}\right)= & 1+l_{2}\left(l_{1}+1\right)^{2}-\frac{1}{3} l_{1}\left(l_{1}^{2}-4\right)+\frac{1}{24}|\Delta l|\left(\Delta l^{2}-4\right)-\frac{1}{4} \Delta l^{2}\left(l_{1}+1\right)(3 \\
& +\frac{1}{6} q\left(q^{2}-1\right)-\frac{1}{3} m_{1}\left(m_{1}-1\right)\left(m_{1}-2\right)-\frac{1}{3} m_{2}\left(m_{2}-1\right)\left(m_{2}-2\right),
\end{aligned}
$$


where $\Delta l=l_{3}-\bar{l}_{3}$ and

$$
q=\max \left(0, \frac{1}{2}|\Delta l|+l_{1}-l_{2}\right), m_{1}=\max \left(0, \frac{1}{2}|\Delta l|-l_{1}\right), m_{2}=\max \left(0, \frac{1}{2}|\Delta l|-l_{2}\right) .
$$

The domain of validity of this formula is $l_{1} \leq l_{2}$ and $l_{1}+l_{2}-\frac{1}{2}|\Delta l| \leq \min \left(l_{3}, \bar{l}_{3}\right)$. When $\bar{l}_{3}=l_{3}$ eq. (3.38) agrees with the analytic counting of independent tensor structures performed in ref. [9]..$^{3}$

In principle the classification performed here for three-point functions can be extended to four (or higher) point functions, although its complexity rapidly grows. There are 64 $\mathrm{SU}(2,2)$ invariant building blocks (compared to 15 for three-point functions) and many relations among bi-products of invariants for four-point functions. For this reason we have not attempted to make a general classification of correlators with more than three fields.

\section{4 $6 \mathrm{D}$ to $4 \mathrm{D}$ dictionary}

The transition from the $6 \mathrm{D}$ index-free form to the $4 \mathrm{D}$ one is extremely easy. Given a $6 \mathrm{D}$ three-point function, we just need to rewrite the invariants $I, K, \bar{K}, J$ in $4 \mathrm{D}$ form. We have

$$
\begin{aligned}
& I_{i j}=\frac{1}{X_{i}^{+} X_{j}^{+}} \bar{s}_{i \dot{\alpha}}\left(\overline{\mathbf{X}}_{i} \mathbf{X}_{j}\right)_{\alpha}^{\dot{\alpha}} s_{j}^{\alpha}=\bar{s}_{i \dot{\alpha}}\left(x_{i j} \cdot \sigma \epsilon\right)_{\alpha}^{\dot{\alpha}} s_{j}^{\alpha}, \\
& K_{k, i j}=\frac{N_{k, i j}}{X_{i}^{+} X_{j}^{+}} s_{i}^{\alpha} s_{j}^{\beta}\left(\mathbf{X}_{i} \overline{\mathbf{X}}_{k} \mathbf{X}_{j}\right)_{\alpha \beta} \\
& =\frac{i}{\sqrt{2}} \frac{\left|x_{i j}\right|}{\left|x_{i k}\right|\left|x_{j k}\right|} s_{i}^{\alpha} s_{j}^{\beta}\left(\left(x_{i k}^{2}+x_{j k}^{2}-x_{i j}^{2}\right) \epsilon_{\alpha \beta}+4 x_{i k}^{\mu} x_{k j}^{\nu}\left(\sigma_{\mu \nu} \epsilon\right)_{\alpha \beta}\right), \\
& \bar{K}_{k, i j}=\frac{N_{k, i j}}{X_{i}^{+} X_{j}^{+}} \bar{s}_{i \dot{\alpha}} \bar{s}_{j \dot{\beta}}\left(\overline{\mathbf{X}}_{i} \mathbf{X}_{k} \overline{\mathbf{X}}_{j}\right)^{\dot{\alpha} \dot{\beta}} \\
& =\frac{i}{\sqrt{2}} \frac{\left|x_{i j}\right|}{\left|x_{i k}\right|\left|x_{j k}\right|} \bar{s}_{i \dot{\alpha}} \bar{s}_{j \dot{\beta}}\left(\left(x_{i k}^{2}+x_{j k}^{2}-x_{i j}^{2}\right) \epsilon^{\dot{\alpha} \dot{\beta}}+4 x_{i k}^{\mu} x_{k j}^{\nu}\left(\bar{\sigma}_{\mu \nu} \epsilon\right)^{\dot{\alpha} \dot{\beta}}\right), \\
& J_{k, i j}=\frac{N_{i j}}{\left(X_{k}^{+}\right)^{2}} \bar{s}_{k \dot{\alpha}}\left(\overline{\mathbf{X}}_{k} \mathbf{X}_{i} \overline{\mathbf{X}}_{j} \mathbf{X}_{k}\right)_{\alpha}^{\dot{\alpha}} s_{k}^{\alpha}=2 \frac{x_{i k}^{2} x_{k j}^{2}}{x_{i j}^{2}} s_{k}^{\alpha}\left(Z_{k, i j} \cdot \sigma \epsilon\right)_{\alpha}^{\dot{\alpha}} \bar{s}_{k \dot{\alpha}},
\end{aligned}
$$

where

$$
Z_{k, i j}^{\mu} \equiv \frac{x_{k i}^{\mu}}{x_{k i}^{2}}-\frac{x_{k j}^{\mu}}{x_{k j}^{2}}, \quad Z_{k, i j}^{\mu}=-Z_{k, j i}^{\mu} .
$$

Explicit 4D correlation functions with indices are obtained by removing the auxiliary spinors $s_{i}$ and $\bar{s}_{i}$ through derivatives, as described in eq. (2.16).

\subsection{Transformations under 4D parity}

Under the 4D parity transformation $\left(x^{0}, \vec{x}\right) \rightarrow\left(x^{0},-\vec{x}\right)$, a 4D field in the $(l, \bar{l})$ representation of the Lorentz group is mapped to a field in the complex conjugate representation $(\bar{l}, l)$. We parametrize the transformation as follows:

$$
f_{\alpha_{1} \ldots \alpha_{l}}^{\dot{\beta}_{1} \ldots \dot{\beta}_{\bar{l}}}(x) \rightarrow \eta(-)^{\frac{l+\bar{l}}{2}} f_{\beta_{1} \ldots \beta_{\bar{l}}}^{\dot{\alpha}_{1} \ldots \dot{\alpha}_{l}}(\tilde{x})
$$

\footnotetext{
${ }^{3}$ When matching our result with ref. [9] one should not forget that eq. (3.38) counts both parity-even and parity-odd structures.
} 
where $\eta$ is the intrinsic parity of the field and $\tilde{x}$ is the parity transformed coordinate. Applying parity twice gives

$$
\eta \eta_{c}(-)^{l+\bar{l}}=1
$$

where $\eta_{c}$ is the intrinsic parity of the conjugate field. We then see that $\eta \eta_{c}=+1$ for bosonic operators and $\eta \eta_{c}=-1$ for fermionic ones. Under parity, in particular, we have

$$
(x \cdot \sigma \epsilon)_{\alpha}^{\dot{\beta}} \leftrightarrow-(x \cdot \bar{\sigma} \epsilon)_{\beta}^{\dot{\alpha}}, \quad \epsilon_{\alpha \beta} \leftrightarrow-\epsilon^{\dot{\alpha} \dot{\beta}}, \quad x^{\mu} y^{\nu}\left(\sigma_{\mu \nu} \epsilon\right)_{\alpha \beta} \leftrightarrow-x^{\mu} y^{\nu}\left(\bar{\sigma}_{\mu \nu} \epsilon\right)^{\dot{\alpha} \dot{\beta}} .
$$

We can see how parity acts on the $6 \mathrm{D}$ invariants (3.11)-(3.14) by using their $4 \mathrm{D}$ expressions (3.40)-(3.43) on the null cone and eqs. (3.47). We get

$$
\begin{aligned}
I_{i j} & \rightarrow-I_{j i}, \\
K_{i, j k} & \rightarrow+\bar{K}_{i, j k}, \\
\bar{K}_{i, j k} & \rightarrow+K_{i, j k}, \\
J_{i, j k} & \rightarrow+J_{i, j k} .
\end{aligned}
$$

In general, parity maps correlators of fields into correlators of their complex conjugate fields. Imposing parity in a CFT implies that for each primary field $(l, \bar{l})$ there must exist its conjugate one $(\bar{l}, l)$, and the constants entering in their correlators are related. Of course, we can also have correlators that are mapped to themselves under parity. Since $\Delta l \rightarrow-\Delta l$ under parity, where $\Delta l$ is defined in eq. (3.32), such correlators should have $\Delta l=0$. Due to eqs. (3.34) and (3.28), the structures $K$ and $\bar{K}$ cannot enter in correlators with $\Delta l=0$, which depend only on the invariants $I_{i j}$ and $J_{i, j k}$. For correlators that are mapped to themselves under parity, one has to take linear combinations of the tensor structures appearing in eq. (3.31) that are even or odd under parity, according to the transformation rules for I's and $J$ 's in eq. (3.48). Depending on the intrinsic parity of the product of the fields entering the correlator, the coefficients multiplying the parity even or parity odd structures should then be set to zero if parity is conserved.

A particular relevant class of correlators that are mapped to themselves under parity are those involving symmetric traceless tensors only. In this case we have verified that eqs. (3.48) lead to the correct number of parity even and parity odd structures as separately computed in ref. [9].

\section{Conserved operators}

Primary tensor fields whose scaling dimension $\Delta$ saturates the unitarity bound [27] (see also ref. [28] for a generalization to $D \neq 4$ space-time dimensions)

$$
\Delta \geq \frac{l+\bar{l}}{2}+2, \quad l \neq 0 \text { and } \bar{l} \neq 0,
$$

are conserved. Three-point functions with conserved operators are subject to further constraints which will be analyzed in this section. Given a conserved spinor-tensor primary field in the $(l, \bar{l})$ representation of the Lorentz group, with scaling dimension $\Delta$, we define

$$
(\partial \cdot f)_{\alpha_{2} \ldots \alpha_{l}}^{\dot{\beta}_{2} \ldots \dot{\beta}_{\bar{l}}}(x) \equiv\left(\epsilon \sigma^{\mu}\right)_{\dot{\beta}_{1}}^{\alpha_{1}} \partial_{\mu} f_{\alpha_{1} \ldots \alpha_{l}}^{\dot{\beta}_{1} \ldots \dot{\beta}_{\bar{l}}}(x)=0 .
$$


Let us see how the 4D current conservation (4.2) can be uplifted to $6 \mathrm{D}$ as a constraint on the field $F_{b_{1} \ldots b_{\bar{l}}}^{a_{1} \ldots a_{l}}$. This will allow us to work directly with the 6D invariants (3.11)-(3.14), providing a great simplification. The analysis that follows is essentially a generalization to arbitrary conserved currents of the one made in ref. [9], where only symmetric traceless currents were considered. From eq. (2.10), we get

$$
(\partial \cdot f)_{\alpha_{2} \ldots \alpha_{l}}^{\dot{\beta}_{2} \ldots \dot{\beta}_{\bar{l}}}(x)=\left(X^{+}\right)^{\Delta-(l+\bar{l}) / 2} \partial_{\mu}\left(\left(e^{\mu}\right)_{a_{1}}^{b_{1}} \mathbf{X}_{\alpha_{2} a_{2}} \ldots \mathbf{X}_{\alpha_{l} a_{l}} \overline{\mathbf{X}}^{\dot{\beta}_{2} b_{2}} \ldots \overline{\mathbf{X}}^{\dot{\beta}_{\bar{l}} b_{\bar{l}}} F_{b_{1} \ldots b_{\bar{l}}}^{a_{1} \ldots a_{l}}(X)\right)
$$

where

$$
\left(e^{\mu}\right)_{a}^{b} \equiv-\mathbf{X}_{a \alpha}\left(\epsilon \sigma^{\mu}\right)_{\dot{\beta}}^{\alpha} \overline{\mathbf{X}}^{\dot{\beta} b}=\left(M^{\mu+}\right)_{a}^{b},
$$

in terms of the tensor

$$
M^{M N}=2\left(X^{M} \Sigma_{P}^{N} X^{P}-X^{N} \Sigma_{P}^{M} X^{P}\right) .
$$

Applying the derivative to each term gives

$$
\begin{aligned}
(\partial \cdot f)_{\alpha_{2} \ldots \alpha_{l}}^{\dot{\beta}_{2} \ldots \dot{\beta}_{\bar{l}}} & =\left(X^{+}\right)^{\Delta-(l+\bar{l}) / 2} \mathbf{X}_{\alpha_{3} a_{3}} \ldots \mathbf{X}_{\alpha_{l} a_{l}} \overline{\mathbf{X}}^{\dot{\beta}_{3} b_{3}} \ldots \overline{\mathbf{X}}^{\dot{\beta}_{\bar{l}} b_{\bar{l}}}\left(\left(\partial_{\mu} e^{\mu}\right)_{a_{1}}^{b_{1}} \mathbf{X}_{\alpha_{2} a_{2}} \overline{\mathbf{X}}^{\dot{\beta}_{2} b_{2}}+\frac{\partial X^{M}}{\partial x^{\nu}}\left(e^{\nu}\right)_{a_{1}}^{b_{1}}\right. \\
& \left.\times\left((l-1)\left(\frac{\partial \mathbf{X}_{\alpha_{2} a_{2}}}{\partial X^{M}}\right) \overline{\mathbf{X}}^{\dot{\beta}_{2} b_{2}}+(\bar{l}-1)\left(\frac{\partial \overline{\mathbf{X}}^{\dot{\beta}_{2} b_{2}}}{\partial X^{M}}\right) \mathbf{X}_{\alpha_{2} a_{2}}+\mathbf{X}_{\alpha_{2} a_{2}} \overline{\mathbf{X}}^{\dot{\beta}_{2} b_{2}} \frac{\partial}{\partial X^{M}}\right)\right) F_{b_{1} \ldots b_{\bar{l}}}^{a_{1} \ldots a_{l}}
\end{aligned}
$$

After some algebraic manipulations, eq. (4.6) can be recast in the form

$$
(\partial \cdot f)_{\alpha_{2} \ldots \alpha_{l}}^{\dot{\beta}_{2} \ldots \dot{\beta}_{\bar{l}}}=\left(X^{+}\right)^{\Delta-(l+\bar{l}) / 2+2} \mathbf{X}_{\alpha_{2} a_{2}} \ldots \mathbf{X}_{\alpha_{l} a_{l}} \overline{\mathbf{X}}^{\dot{\beta}_{2} b_{2}} \ldots \overline{\mathbf{X}}^{\dot{\beta}_{l} b_{\bar{l}}} R_{b_{2} \ldots b_{\bar{l}}}^{a_{2} \ldots a_{l}}
$$

where

$$
R_{b_{2} \ldots b_{\bar{l}}}^{a_{2} \ldots a_{l}}=2\left(-\left(X_{M} \Sigma^{M N} \frac{\partial}{\partial X^{N}}\right)_{a_{1}}^{b_{1}}+\frac{1}{X^{+}}\left(\Delta-\frac{l+\bar{l}}{2}-2\right) X_{M}\left(\Sigma^{M+}\right)_{a_{1}}^{b_{1}}\right) F_{b_{1} \ldots b_{\bar{l}}}^{a_{1} \ldots a_{l}} .
$$

In writing eq. (4.8), we used the fact that $F$ is a homogeneous function of degree $\Delta+(l+\bar{l}) / 2$ and the following two identities hold:

$$
\left(\left(X_{M} \Sigma^{M N} \frac{\partial}{\partial X^{N}}\right)_{a_{1}}^{b_{1}} \mathbf{X}_{\alpha_{2} a_{2}}\right) F_{b_{1} \ldots b_{\bar{l}}}^{a_{1} \ldots a_{l}}=\left(\left(X_{M} \Sigma^{M N} \frac{\partial}{\partial X^{N}}\right)_{a_{1}}^{b_{1}} \overline{\mathbf{X}}^{\dot{\beta}_{2} b_{2}}\right) F_{b_{1} \ldots b_{\bar{l}}}^{a_{1} \ldots a_{l}}=0
$$

since $F$ is symmetric in its indices and satisfies eq. (2.11).

Analogously to what found in ref. [9] for symmetric traceless operators, we see here what is special about operators that saturate the unitarity bound (4.1). They are the only ones for which the $6 \mathrm{D}$ uplifted tensor $R$ is $\mathrm{SO}(4,2)$ covariant. In our index-free notation, current conservation in $6 \mathrm{D}$ takes an extremely simple form:

$$
\partial \cdot f(x, s, \bar{s})=(\partial \cdot f(x))_{\alpha_{2} \ldots \alpha_{l}}^{\dot{\beta}_{2} \ldots \dot{\beta}_{\bar{l}}} s^{\alpha_{2}} \ldots s^{\alpha_{l}} \bar{s}_{\dot{\beta}_{2}} \ldots \bar{s}_{\dot{\beta}_{\bar{l}}}=-2 D \cdot F(X, S, \bar{S})=0,
$$

where

$$
D=\left(X_{M} \Sigma^{M N} \frac{\partial}{\partial X^{N}}\right)_{a}^{b} \frac{\partial}{\partial S_{a}} \frac{\partial}{\partial \bar{S}^{b}}
$$




\section{Example: fermion-fermion-tensor correlator}

In this section we show some examples on how to use the formalism presented in section 3. In particular, we will determine all the three-point functions involving two fermion fields $\psi_{\alpha}$ and $\bar{\chi}^{\dot{\beta}}:\left\langle\psi_{\alpha}\left(x_{1}\right) \bar{\chi}^{\dot{\beta}}\left(x_{2}\right) \mathcal{O}\left(x_{3}\right)\right\rangle{ }^{4}$ According to eq. (3.34), the only non-vanishing 3-point function occurs when $\mathcal{O}$ is in one of the following three Lorentz representations: $(l, l)$, $(l+2, l)$ and $(l, l+2)$, with $l \geq 0$. We will determine the form of the correlators in two cases: with non-conserved and conserved operator $\mathcal{O}$.

\subsection{Non-conserved tensor}

Let us start by considering the $(l, l)$ representations. According to eq. (3.31), for $l=0$ there is only one possible structure to this correlator, proportional to $I_{21}$. Using eqs. (2.16) and (3.40) we immediately get

$$
\left\langle\psi_{\alpha}\left(x_{1}\right) \bar{\chi}^{\dot{\beta}}\left(x_{2}\right) \phi\left(x_{3}\right)\right\rangle=\lambda_{\psi \bar{\chi} \mathcal{O}_{0,0}} x_{12}^{-\Delta_{123}-1} x_{13}^{-\Delta_{132}} x_{23}^{-\Delta_{231}}\left(x_{21} \cdot \sigma \epsilon\right)_{\alpha}^{\dot{\beta}},
$$

with $\lambda_{\psi \bar{\chi}} \mathcal{O}_{0,0}$ a complex parameter. For $l \geq 1$, two independent structures are present,

$$
\left\langle F_{1} F_{2} F_{3}\right\rangle=\mathcal{K}\left(\lambda_{1} I_{21} J_{3,12}+\lambda_{2} I_{31} I_{23}\right) J_{3,12}^{l-1}, \quad l \geq 1,
$$

where $\lambda_{1,2}$ are two complex parameters and $\mathcal{K}$ is defined in eq. (3.2). Again using eqs. (2.16) and (3.40) we find

$$
\begin{aligned}
\left\langle\psi_{\alpha}\left(x_{1}\right) \bar{\chi}^{\dot{\beta}}\left(x_{2}\right) t_{\alpha_{1} \ldots \alpha_{l}}^{\dot{\beta}_{1} \ldots \dot{\beta}_{l}}\left(x_{3}\right)\right\rangle=\frac{x_{12}^{-\Delta_{123}-l-1} x_{13}^{-\Delta_{132}+l} x_{23}^{-\Delta_{231}+l}}{(l !)^{2}} \times \\
\quad \times\left(\lambda_{\psi \bar{\chi} \mathcal{O}_{l, l}}^{(1)}\left(x_{12} \cdot \sigma \epsilon\right)_{\alpha}^{\dot{\beta}}\left(Z_{3,12} \cdot \sigma \epsilon\right)_{\alpha_{1}}^{\dot{\beta}_{1}} \ldots\left(Z_{3,12} \cdot \sigma \epsilon\right)_{\alpha_{l}}^{\dot{\beta}_{l}}+\frac{x_{12}^{2}}{2 x_{13}^{2} x_{23}^{2}}\right. \\
\quad \times \lambda_{\psi \bar{\chi} \mathcal{O}_{l, l}}^{(2)}\left(x_{13} \cdot \sigma \epsilon\right)_{\alpha}^{\dot{\beta}_{1}}\left(x_{23} \cdot \sigma \epsilon\right)_{\alpha_{1}}^{\dot{\beta}}\left(Z_{3,12} \cdot \sigma \epsilon\right)_{\alpha_{2}}^{\dot{\beta}_{2}} \ldots\left(Z_{3,12} \cdot \sigma \epsilon\right)_{\alpha_{l}}^{\dot{\beta}_{l}} \\
\quad+\text { perms. }) .
\end{aligned}
$$

In eq. (5.3), $\lambda_{\psi \bar{\chi} \mathcal{O}_{l, l}}^{(1)}$ and $\lambda_{\psi \bar{\chi} \mathcal{O}_{l, l}}^{(2)}$ are proportional to $\lambda^{1}$ and $\lambda^{2}$ in eq. (5.2) respectively with the same proportionality factor, $Z_{3,12}^{\mu}$ is defined in eq. (3.44) and perms. refer to the $(l !)^{2}-1$ terms obtained by permuting the $\alpha_{i}$ and $\dot{\beta}_{i}$ indices. When $\bar{\chi}$ is the complex conjugate of $\psi$, namely $\bar{\chi}^{\dot{\beta}}=\bar{\psi}^{\dot{\beta}}=\left(\psi_{\beta}\right)^{\dagger}$ and the symmetric traceless tensor components are real, the OPE coefficients $\lambda_{\psi \bar{\chi} t_{l}}^{(1,2)}$ are either purely real or purely imaginary, depending on $l$. When $x_{1,2,3}^{\mu}$ are space-like separated, causality implies that the operators commute between each other [12]. Taking $\beta=\alpha$ and $\beta_{i}=\alpha_{i}$, we then have

$$
\left\langle\psi_{\alpha}\left(x_{1}\right) \bar{\psi}^{\dot{\alpha}}\left(x_{2}\right) t_{\alpha_{1} \ldots \alpha_{l}}^{\dot{\alpha}_{1} \ldots \dot{\alpha}_{l}}\left(x_{3}\right)\right\rangle^{*}=-\left\langle\psi_{\alpha}\left(x_{2}\right) \bar{\psi}^{\dot{\alpha}}\left(x_{1}\right) t_{\alpha_{1} \ldots \alpha_{l}}^{\dot{\alpha}_{1} \ldots \dot{\alpha}_{l}}\left(x_{3}\right)\right\rangle .
$$

${ }^{4}$ Three-point functions between two fermions and i) one scalar, ii) one vector, one rank two iii) symmetric or iv) antisymmetric tensor have already been considered in appendix B of ref. [29], though some tensor structures in the correlators were missed in that paper. 
Since $Z_{3,12}=-Z_{3,21}$ we get

$$
\left(\lambda_{\psi \bar{\psi} \mathcal{O}_{l, l}}^{(1)}\right)^{*}=(-1)^{l} \lambda_{\psi \bar{\psi} \mathcal{O}_{l, l}}^{(1)}, \quad\left(\lambda_{\psi \bar{\psi} \mathcal{O}_{l, l}}^{(2)}\right)^{*}=(-1)^{l} \lambda_{\psi \bar{\psi} \mathcal{O}_{l, l}}^{(2)}
$$

Let us now consider the parity transformations of eq. (5.3). Parity maps the threepoint function $\left\langle\psi_{\alpha}\left(x_{1}\right) \bar{\chi}^{\dot{\beta}}\left(x_{2}\right) t_{\alpha_{1} \ldots \alpha_{l}}^{\dot{\beta}_{1} \ldots \dot{\beta}_{l}}\left(x_{3}\right)\right\rangle$ to the complex conjugate three-point function $\left\langle\bar{\psi}^{\dot{\alpha}}\left(\tilde{x}_{1}\right) \chi_{\beta}\left(\tilde{x}_{2}\right) t_{\beta_{1} \ldots \beta_{l}}^{\dot{\alpha}_{1} \ldots \dot{\alpha}_{l}}\left(\tilde{x}_{3}\right)\right\rangle$. When $\bar{\chi}=\bar{\psi}$, and $\alpha_{i}=\beta_{i}$, the three-point function is mapped to itself, provided the exchange $x_{1} \leftrightarrow x_{2}$ and $\alpha \leftrightarrow \beta$. The two structures appearing in eq. (5.3) have the same parity transformations. If we impose parity conservation in the CFT and we choose a negative intrinsic parity for the traceless symmetric tensor, $\eta_{\mathcal{O}}=-1$, then the three-point function must vanish: $\lambda_{\psi \bar{\psi} t_{l}}^{(1)}=\lambda_{\psi \bar{\psi} t_{l}}^{(2)}=0$. For $\eta_{\mathcal{O}}=1$, instead, parity invariance does not give any constraint.

Let us next consider the $(l+2, l)$ representations. According to eq. (3.31), there is only one possible structure to this correlator, for any $l$ :

$$
\left\langle F_{1} F_{2} F_{3}\right\rangle=\mathcal{K} \lambda I_{23} K_{2,13} J_{3,12}^{l}
$$

that gives rise to the $4 \mathrm{D}$ correlator

$$
\begin{aligned}
\left\langle\psi_{\alpha}\left(x_{1}\right) \bar{\chi}^{\dot{\beta}}\left(x_{2}\right) t_{\alpha_{1} \ldots \alpha_{l+2}}^{\dot{\beta}_{1} \ldots \dot{\beta}_{l}}\left(x_{3}\right)\right\rangle=\frac{x_{12}^{-\Delta_{123}-l-1} x_{13}^{-\Delta_{132}+l} x_{23}^{-\Delta_{231}+l-2}}{(l !)(l+2) !} \lambda_{\psi} \mathcal{O}_{l+2, l}\left(\left(x_{23} \cdot \sigma \epsilon\right)_{\alpha_{l+1}}^{\dot{\beta}} \times\right. \\
\quad \times\left(\left(x_{12}^{2}+x_{23}^{2}-x_{13}^{2}\right) \epsilon_{\alpha \alpha_{l+2}}+4 x_{12}^{\mu} x_{23}^{\nu}\left(\sigma_{\mu \nu} \epsilon\right)_{\alpha \alpha_{l+2}}\right) \times \\
\left.\quad \times\left(Z_{3,12} \cdot \sigma \epsilon\right)_{\alpha_{1}}^{\dot{\beta}_{1}} \ldots\left(Z_{3,12} \cdot \sigma \epsilon\right)_{\alpha_{l}}^{\dot{\beta}_{l}}+\text { perms. }\right)
\end{aligned}
$$

where $\lambda_{\psi \bar{\chi} \mathcal{O}}$ is proportional to $\lambda$ in eq. (5.6).

A similar analysis applies to the complex conjugate $(l, l+2)$ representations. The only possible $6 \mathrm{D}$ structure is

$$
\left\langle F_{1} F_{2} F_{3}\right\rangle=\mathcal{K} \lambda I_{31} \bar{K}_{1,23} J_{3,12}^{l}
$$

and gives

$$
\begin{aligned}
\left\langle\psi_{\alpha}\left(x_{1}\right)\right. & \left.\bar{\chi}^{\dot{\beta}}\left(x_{2}\right) t_{\alpha_{1} \ldots \alpha_{l}}^{\dot{\beta}_{1} \ldots \dot{\beta}_{l+2}}\left(x_{3}\right)\right\rangle=\frac{x_{12}^{-\Delta_{123}-l-1} x_{13}^{-\Delta_{132}+l-2} x_{23}^{-\Delta_{231}+l}}{(l !)(l+2) !} \lambda_{\psi \bar{\chi} \mathcal{O}_{l, l+2}}\left(\left(x_{31} \cdot \sigma \epsilon\right)_{\alpha}^{\dot{\beta}_{l+1}} \times\right. \\
& \times\left(\left(x_{12}^{2}+x_{13}^{2}-x_{23}^{2}\right) \epsilon^{\dot{\beta} \dot{\beta}_{l+2}}+4 x_{21}^{\mu} x_{13}^{\nu}\left(\bar{\sigma}_{\mu \nu} \epsilon\right)^{\dot{\beta} \dot{\beta}_{l+2}}\right) \times \\
& \left.\times\left(Z_{3,12} \cdot \sigma \epsilon\right)_{\alpha_{1}}^{\dot{\beta}_{1}} \ldots\left(Z_{3,12} \cdot \sigma \epsilon\right)_{\alpha_{l}}^{\dot{\beta}_{l}}+\text { perms. }\right)
\end{aligned}
$$

If $\chi=\psi$, as expected, eq. (5.9) is mapped to eq. (5.7) under parity transformation. In particular, in a parity invariant CFT, we should have the same number of $(l, l+2)$ and conjugate $(l+2, l)$ fields, with

$$
\lambda_{\psi \bar{\psi} \mathcal{O}_{l+2, l}}=\eta_{\mathcal{O}} \lambda_{\psi \bar{\psi} \mathcal{O}_{l, l+2}}
$$




\subsection{Conserved tensor}

Let us start by considering $(l, l)$ representations. The scaling dimension of $\mathcal{O}$ is now fixed to be $\Delta_{3}=l+2$. Taking the divergence (4.11) of eq. (5.2) and using eqs. (2.15) and (A.13) gives

$$
D_{3} F=\frac{\Delta_{1}-\Delta_{2}}{2} \frac{2 \lambda_{2}(l-1)(l+2) I_{31} I_{23} J_{3,12}^{l-2}+\left[\lambda_{2}+2 l(l+1) \lambda_{1}\right] I_{21} J_{3,12}^{l-1}}{X_{12}^{\frac{\Delta_{1}+\Delta_{2}-2 l-1}{2}} X_{13}^{\frac{\Delta_{1}-\Delta_{2}+2 l+2}{2}} X_{23}^{\frac{-\Delta_{1}+\Delta_{2}+2 l+2}{2}}}=0,
$$

where the subscript 3 in $D$ indicates that derivatives are taken with respect to $X_{3}, S_{3}$ and $\bar{S}_{3}$. Eq. (5.11) has the correct form for a Fermion-Fermion-spin $(l-1)$ symmetric tensor, as it should, and is automatically satisfied if $\Delta_{1}=\Delta_{2}$. For $\Delta_{1} \neq \Delta_{2}$ we have

$$
2 \lambda_{2}(l-1)(l+2)=0, \quad \lambda_{1}=-\frac{\lambda_{2}}{2 l(l+1)} .
$$

For $l=1$ we get one independent structure in eq. (5.2) with $\lambda_{2}=-4 \lambda_{1}$. For $l>1$ eq. (5.12) admits only the trivial solution

$$
\left\langle\psi_{\alpha}\left(x_{1}\right) \bar{\chi}^{\dot{\beta}}\left(x_{2}\right) \mathcal{O}_{\alpha_{1} \ldots \alpha_{l}}^{\dot{\beta}_{1} \ldots \dot{\beta}_{l}}\left(x_{3}\right)\right\rangle=0, \quad l>1, \quad \Delta_{1} \neq \Delta_{2} .
$$

Let us next consider the $(l+2, l)$ representations, where $\mathcal{O}^{(l+2, l)}$ is a conserved tensor with $\Delta_{3}=l+3, l>0$. The divergence (4.11) of eq. (5.6) gives now

$$
D_{3} F=-\frac{\lambda}{2}\left(\Delta_{2}-\Delta_{1}+1\right) \frac{2 l(l+3) I_{23} K_{2,31} J_{3,12}^{l-1}}{X_{12}^{\frac{\Delta_{1}+\Delta_{2}-2 l-3}{2}} X_{13^{\frac{\Delta_{1}-\Delta_{2}+2 l+4}{2}}}^{X_{23}^{\frac{-\Delta_{1}+\Delta_{2}+2 l+4}{2}}}}=0 .
$$

For $\Delta_{2}=\Delta_{1}-1$ eq. (5.14) is automatically satisfied. When $\Delta_{2} \neq \Delta_{1}-1$, there are no non-trivial solutions of eq. (5.14) for $l>0$ :

$$
\left\langle\psi_{\alpha}\left(x_{1}\right) \bar{\chi}^{\dot{\beta}}\left(x_{2}\right) \mathcal{O}_{\alpha_{1} \ldots \alpha_{l+2}}^{\dot{\beta}_{1} \ldots \dot{\beta}_{l}}\left(x_{3}\right)\right\rangle=0, \quad l>0, \quad \Delta_{2} \neq \Delta_{1}-1 .
$$

A similar result applies for conserved $\mathcal{O}^{(l, l+2)}$ operators.

We have checked that the current conservation condition (4.10) reproduces various results found in the literature. In particular we have verified that the correlator of three energy-momentum tensors, once permutations and current conservations are imposed, contains three independent structures, as found in ref. [1].

\section{Consistency with crossing symmetry: counting four-point function structures}

We have seen how three-point functions of spinor-tensors in arbitrary representations of the Lorentz group can be computed. The most subtle step of the procedure is the identification of the independent tensor structures entering three-point functions. For the particular case of traceless symmetric tensors, we reproduce the results of ref. [9]. But only a subset of the building blocks we have found enter traceless symmetric tensors, so more checks are welcome. In this section we use four-point functions to show how our three-point function counting passes the highly non-trivial consistency check of crossing 
symmetry. Recall that, by using the OPE, the number of independent structures $N_{4}$ entering a generic four-point function

$$
\left\langle\mathcal{O}_{1}\left(x_{1}\right) \mathcal{O}_{2}\left(x_{2}\right) \mathcal{O}_{3}\left(x_{3}\right) \mathcal{O}_{4}\left(x_{4}\right)\right\rangle
$$

can be put in one to one correspondence with those of the three-point functions. For instance by taking the limit $x_{1} \rightarrow x_{2}$ and $x_{3} \rightarrow x_{4}$, eq. (6.1) schematically boils down to the sum of the two-point functions

$$
\sum_{r} \sum_{i, j} C_{\mathcal{O}_{1} \mathcal{O}_{2} \mathcal{O}_{r}}^{i} C_{\mathcal{O}_{3} \mathcal{O}_{4} \mathcal{O}_{\bar{r}}}^{j}\left\langle\mathcal{O}_{r}^{i}\left(x_{2}\right) \mathcal{O}_{\bar{r}}^{j}\left(x_{4}\right)\right\rangle
$$

In eq. (6.2), $r$ runs over all possible representations that can appear simultaneously in the two OPE's ( $\bar{r}$ being the complex conjugate one), while $i$ and $j$ denote, for a given representation $r$, the possible independent OPE coefficients, one for each independent tensor structure. All kinematic factors and tensor structures have been omitted for simplicity. Denoting the number of structures in the three-point function $\left\langle\mathcal{O}_{i} \mathcal{O}_{j} \mathcal{O}_{r}\right\rangle$ by $N_{3 r}^{i j}$, we conclude that

$$
N_{4}=\sum_{r} N_{3 r}^{12} N_{3 \bar{r}}^{34}
$$

On the other hand, it is clear that the very same number $N_{4}$ should be obtained by pairing the four operators in any other way:

$$
\sum_{r} N_{3 r}^{12} N_{3 \bar{r}}^{34}=\sum_{r} N_{3 r}^{14} N_{3 \bar{r}}^{23}=\sum_{r} N_{3 r}^{13} N_{3 \bar{r}}^{24}
$$

We will refer to the three OPE pairing in eq. (6.4) as the $s, t$ or $u$-channel, respectively. We have numerically verified the validity of eq. (6.4) for any four-point function involving arbitrary non-conserved fermionic or bosonic operators with $0 \leq l_{i}, \bar{l}_{i} \leq 6, i=1, \ldots 4$. Finding a closed analytic form of $N_{3 r}^{i j}$ in the most general case is a laborious task, so we focus here on the case in which the external operators are all symmetric traceless. The number of independent structures appearing in the three-point function of two symmetric traceless operators $\left(l_{1}, l_{1}\right)\left(l_{2}, l_{2}\right)$ and one arbitrary $\left(l_{x}, \bar{l}_{x}\right)$ tensor has been found in eq. (3.38). Using that formula and summing over all the possible representations that can be exchanged, we can obtain in a closed analytical form the number of independent structures for any four-point function involving arbitrary non-conserved symmetric traceless operators.

For simplicity, let us consider four symmetric traceless operators with $l_{3}=l_{1}, l_{4}=l_{2}$, and $l_{1} \leq l_{2}$. In the $u$-channel, the representations that can appear in the OPE are of the form $\left(l_{x}, l_{x}+\delta\right)$, with $|\delta| \leq 4 l_{1}$. In the $s$ and $t$-channel, they are of the form $\left(l_{x}, l_{x}+\delta\right)$, with $|\delta| \leq 2\left(l_{1}+l_{2}\right)$. Using eq. (3.38) and summing over all the representations exchanged, 
we get the number of four-point function structures. For $l_{2} \leq 2 l_{1}$ we get

$$
\begin{aligned}
N_{4}^{l_{2} \leq 2 l_{1}}\left(l_{1}, l_{2}\right)= & \frac{1}{630}\left(630-35 l_{1}^{7}+1518 l_{2}+1232 l_{2}^{2}+364 l_{2}^{3}+35 l_{2}^{4}+7 l_{2}^{5}\right. \\
& -7 l_{2}^{6}+l_{2}^{7}+7 l_{1}^{6}\left(34 l_{2}-1\right)+l_{1}^{5}\left(175+84 l_{2}-672 l_{2}^{2}\right)+ \\
& +35 l_{1}^{4}\left(1-26 l_{2}-12 l_{2}^{2}+28 l_{2}^{3}\right)+70 l_{1}^{3}\left(1+20 l_{2}+64 l_{2}^{2}+40 l_{2}^{3}\right. \\
& \left.-4 l_{2}^{4}\right)+14 l_{1}^{2}\left(88+447 l_{2}+600 l_{2}^{2}+200 l_{2}^{3}-30 l_{2}^{4}+6 l_{2}^{5}\right) \\
& \left.+14 l_{1}\left(120+398 l_{2}+384 l_{2}^{2}+100 l_{2}^{3}-5 l_{2}^{4}+6 l_{2}^{5}-l_{2}^{6}\right)\right) .
\end{aligned}
$$

For $l_{2} \geq 2 l_{1}$ we get

$$
\begin{aligned}
N_{4}^{l_{2} \geq 2 l_{1}}\left(l_{1}, l_{2}\right)= & \frac{1}{210}\left(210+592 l_{1}+448 l_{1}^{2}-126 l_{1}^{3}-175 l_{1}^{4}+133 l_{1}^{5}+147 l_{1}^{6}+31 l_{1}^{7}\right. \\
& \left.-70 l_{2}\left(l_{1}+1\right)^{4}\left(l_{1}\left(2+l_{1}\right)-7\right)+420\left(1+l_{1}\right)^{4} l_{2}^{2}+140 l_{2}^{3}\left(1+l_{1}\right)^{4}\right) .
\end{aligned}
$$

Although it is not obvious from their expressions, eqs. (6.5) and (6.6) always give rise to positive integers numbers, as they should, and agree for $l_{2}=2 l_{1}$. In both cases, we get the same formula by counting structures either in the $s^{-}, t-$, or $u$-channel. We believe this is a highly non-trivial check supporting the validity of our approach. Eqs. (6.5) and (6.6) count the total number of parity even and parity odd structures. ${ }^{5}$ For illustration we also report the individual number of parity even $\left(N_{4+}\right)$ and parity odd $\left(N_{4-}\right)$ structures when $l_{2}=l_{1}=l$, i.e. four traceless symmetric operators with the same spin. We get

$$
\begin{aligned}
& N_{4+}(l)=\frac{(l+1)(l+2)}{630}(315+l(957+l(1361+l(1127+151 l(l+4))))) \\
& N_{4-}(l)=\frac{(l+1) l}{630}(339+l(1789+l(2985+l(2335+151 l(l+6)))))
\end{aligned}
$$

We have in particular $N_{4+}(1)=43, N_{4+}(2)=594, N_{4+}(3)=4174$. Our formula for $N_{4+}(l)$ does not agree with eq. (4.68) of ref. [9] for $l \geq 2$. The number of structures which is found by using eq. (4.68) of that paper is slightly bigger than what found using $N_{4+}$ in eq. (6.7) (there is agreement between the two formulas only for $l=1$ ). The same kind of mismatch is found for four-point functions featuring traceless symmetric operators with different spins. We believe that ref. [9] might have missed some relation between invariants, resulting in an overcounting of structures in four space-time dimensions.

It is important to stress that the number of invariants above refer to the generic case of four different non-conserved operators. For identical operators, the obvious permutation

\footnotetext{
${ }^{5}$ As explained in subsection 3.5, by parity even and odd we mean the structures that are respectively allowed or forbidden when we impose parity conservation to a correlator where the product of the 4 intrinsic parities equals one. In this case, the parity odd structures in vector notation are those involving one $\epsilon_{\mu \nu \rho \sigma}$ tensor.
} 
symmetries should be imposed, resulting in a reduced number of tensor structures. For any given correlation function, the constraints arising from conserved operators are easily worked out using the results of section 4 , but we have not tried to get an analytical general formula in this case.

\section{Conclusions}

We have computed in this paper the most general three point function occurring in a 4D CFT between bosonic and fermionic primary fields in arbitrary representations of the Lorentz group. We have used the 6D embedding formalism in twistor space with an index free notation, as introduced in ref. [13], to efficiently recast the result in terms of $6 \mathrm{D} \mathrm{SU}(2,2)$ invariants. The most important equation of the paper is the compact $6 \mathrm{D}$ formula (3.31), from which any $4 \mathrm{D}$ correlator can easily be extracted. The constraints arising from conserved operators take a very simple form, see eqs. (4.10) and (4.11), and can be solved within our formalism. Once the number of independent tensor structures in three-point functions are known, one can compute the number of independent structures of higher point functions by taking the OPE limit in pairs of operators. As a highly non-trivial check of our results, we have shown that this number is independent of the way the operators are paired in the OPE, as it should be by crossing symmetry.

As one of many applications of our results, we have reported the closed form expression (3.38) for the number of tensor structures in three-point functions of two symmetric traceless and another arbitrary operator. Such result, in turn, allows to analytically determine the number of tensor structures of four-point functions of traceless symmetric tensors, see eqs. (6.5), (6.6) and (6.7).

Understanding three-point functions is the first crucial step to extend the conformal bootstrap beyond scalar four-point functions. The methods used in this paper should allow to determine the conformal blocks associated to fields in arbitrary Lorentz representations entering in arbitrary four-point functions, in terms of a number of "seed" conformal blocks, analogously to the way the results of ref. [9] allow to compute conformal blocks of symmetric traceless tensors entering in four-point functions of symmetric traceless tensors in terms of the known conformal blocks for scalar four-point functions [18]. It would also be nice to extend to arbitrary bosonic and fermionic fields the conjectured agreement that was found between the number of tensor structures in $n$-point functions of symmetric traceless operators in D dimensional CFTs and the number of independent terms in $n$-point scattering amplitudes of massive higher spin particles in flat D +1 dimensional Minkowski space [9]. The embedding twistor formalism developed in this paper should be able to address this point for the $\mathrm{D}=4$ case. We hope to come back to these further applications in a separate publication.

Note added. During the final stages of this work, ref. [14] appeared, where tensors with mixed symmetry are studied. Ref. [14] considers CFTs in arbitrary dimensions, but focuses on bosonic, non-conserved, operators only. When a comparison is possible, the number of tensor structures computed in the examples considered in ref. [14] agrees with our results. ${ }^{6}$

\footnotetext{
${ }^{6}$ We thank Tobias Hansen for some clarifications about the results presented in ref. [14].
} 


\section{Acknowledgments}

We thank Alejandro Castedo Echeverri and Hugh Osborn for useful discussions.

\section{A Notation and conventions}

We follow the conventions of Wess and Bagger [30] (see in particular their appendix A) for the two-component spinor algebra in 4D. Six dimensional vector indices are denoted by $M, N, \ldots$, with $M=\{\mu,+,-\}$; four dimensional vector indices are denoted by $\mu, \nu, \ldots$; four-dimensional spinor indices are denoted by dotted and undotted Greek letters, $\alpha, \beta, \ldots$, $\dot{\alpha}, \dot{\beta}, \ldots$; six-dimensional spinor (twistor) indices are denoted by $a, b, \ldots$, with $a=\{\alpha, \dot{\alpha}\}$. We use capital and small letters for $6 \mathrm{D}$ and $4 \mathrm{D}$ tensors; in particular, $6 \mathrm{D}$ and $4 \mathrm{D}$ coordinates are denoted as $X^{M}$ and $x^{\mu}$, where $x^{\mu}=X^{\mu} / X^{+}$.

The conformal group $\mathrm{SO}(4,2)$ is locally isomorphic to $\mathrm{SU}(2,2)$. The spinorial representations $\boldsymbol{4}_{ \pm}$of $\mathrm{SO}(4,2)$ are mapped to the fundamental and anti-fundamental representations of $\mathrm{SU}(2,2)$. Roughly speaking, $\mathrm{SO}(4,2)$ spinor indices turn into $\mathrm{SU}(2,2)$ twistor indices. We denote by $V_{a}$ and $\bar{W}^{a} \equiv W^{\dagger b} \rho_{b}^{a}$, where $\rho$ is the $\mathrm{SU}(2,2)$ metric, twistors transforming in the fundamental and anti-fundamental of $\mathrm{SU}(2,2)$, respectively:

$$
V \rightarrow U V, \quad \bar{W} \rightarrow \bar{W} \bar{U} .
$$

In eq. (A.1), $U$ and $\bar{U} \equiv \rho U^{\dagger} \rho$ satisfy the condition $\bar{U} U=U \bar{U}=1$.

The non-vanishing components of the $6 \mathrm{D}$ metric $\eta_{M N}$ and its inverse $\eta^{M N}$ in light-cone coordinates are

$$
\eta_{\mu \nu}=\eta^{\mu \nu}=\operatorname{diag}(-1,1,1,1), \quad \eta_{+-}=\eta_{-+}=\frac{1}{2}, \quad \eta^{+-}=\eta^{-+}=2 .
$$

Six dimensional Gamma matrices $\Gamma^{M}$ are constructed by means of the $6 \mathrm{D}$ matrices $\Sigma^{M}$ and $\bar{\Sigma}^{M}$, analogues of $\sigma^{\mu}$ and $\bar{\sigma}^{\mu}$ in 4D:

$$
\Gamma^{M}=\left(\begin{array}{cc}
0 & \Sigma^{M} \\
\bar{\Sigma}^{M} & 0
\end{array}\right),
$$

obeying the commutation relation

$$
\left\{\Gamma^{M}, \Gamma^{N}\right\}=2 \eta^{M N}
$$

It is very useful to choose a basis for the $\Sigma$ and $\bar{\Sigma}$ matrices where they are antisymmetric. This is explicitly given by

$$
\begin{aligned}
\Sigma_{a b}^{M} & =\left\{\left(\begin{array}{cc}
0 & \sigma_{\alpha \dot{\gamma}}^{\mu} \epsilon^{\dot{\beta} \dot{\gamma}} \\
-\bar{\sigma}^{\mu \dot{\alpha} \gamma} \epsilon_{\beta \gamma} & 0
\end{array}\right),\left(\begin{array}{cc}
0 & 0 \\
0 & 2 \epsilon^{\dot{\alpha} \dot{\beta}}
\end{array}\right),\left(\begin{array}{cc}
-2 \epsilon_{\alpha \beta} & 0 \\
0 & 0
\end{array}\right)\right\}, \\
\bar{\Sigma}^{M a c} & =\left\{\left(\begin{array}{cc}
0 & -\epsilon^{\alpha \gamma} \sigma_{\gamma \dot{\beta}}^{\mu} \\
\epsilon_{\dot{\alpha} \dot{\gamma}} \bar{\sigma}^{\mu \dot{\gamma} \beta} & 0
\end{array}\right),\left(\begin{array}{cc}
-2 \epsilon^{\alpha \beta} & 0 \\
0 & 0
\end{array}\right)\left(\begin{array}{cc}
0 & 0 \\
0 & 2 \epsilon_{\dot{\alpha} \dot{\beta}}
\end{array}\right)\right\},
\end{aligned}
$$


where, in order, $M=\{\mu,+,-\}$ in eq. (A.5). The $6 \mathrm{D}$ spinor Lorentz generators are defined as

$$
\begin{aligned}
& \Sigma^{M N}=\frac{1}{4}\left(\Sigma^{M} \bar{\Sigma}^{N}-\Sigma^{N} \bar{\Sigma}^{M}\right), \\
& \bar{\Sigma}^{M N}=\frac{1}{4}\left(\bar{\Sigma}^{M} \Sigma^{N}-\bar{\Sigma}^{N} \Sigma^{M}\right) .
\end{aligned}
$$

Useful relations among the $\Sigma^{M}$ and $\bar{\Sigma}^{M}$ matrices, used repeatedly in the paper, are the following:

$$
\begin{aligned}
\bar{\Sigma}^{M a b} & =-\frac{1}{2} \epsilon^{a b c d} \Sigma_{c d}^{M}, & \Sigma_{a b}^{M} & =-\frac{1}{2} \epsilon_{a b c d} \bar{\Sigma}^{M c d}, \\
\Sigma_{a b}^{M} \Sigma_{M c d} & =2 \epsilon_{a b c d}, & \bar{\Sigma}^{M a b} \bar{\Sigma}_{M}^{c d} & =2 \epsilon^{a b c d}, \\
\Sigma_{a b}^{M} \bar{\Sigma}_{M}^{c d} & =-2\left(\delta_{a}^{c} \delta_{b}^{d}-\delta_{a}^{d} \delta_{b}^{c}\right), & &
\end{aligned}
$$

where $\epsilon_{1234}=\epsilon^{1234}=+1$.

The $6 \mathrm{D}$ null cone is defined by

$$
X^{2}=X^{M} X^{N} \eta_{M N}=0 \Longrightarrow X^{-}=-\frac{X_{\mu} X^{\mu}}{X^{+}} .
$$

On the null cone we have

$$
X_{1} \cdot X_{2}=X_{1}^{M} X_{2}^{N} \eta_{M N}=-\frac{1}{2} X_{1}^{+} X_{2}^{+}\left(x_{1}-x_{2}\right)^{\mu}\left(x_{1}-x_{2}\right)_{\mu},
$$

where $x^{\mu}=X^{\mu} / X^{+}$are the standard $4 \mathrm{D}$ coordinates. We define

$$
x_{i j}^{\mu} \equiv x_{i}^{\mu}-x_{j}^{\mu}, \quad x_{i j}^{2} \equiv x_{i j}^{\mu} x_{\mu, i j} .
$$

Twistor space-coordinates are defined as

$$
\mathbf{X}_{a b} \equiv X_{M} \Sigma_{a b}^{M}=-\mathbf{X}_{b a}, \quad \overline{\mathbf{X}}^{a b} \equiv X_{M} \bar{\Sigma}^{M a b}=-\overline{\mathbf{X}}^{b a} .
$$

A very useful relation is

$$
\mathbf{X} \overline{\mathbf{X}}=X_{M} X_{N} \Sigma^{M} \bar{\Sigma}^{N}=\frac{1}{2} X_{M} X_{N}\left(\Sigma^{M} \bar{\Sigma}^{N}+\Sigma^{N} \bar{\Sigma}^{M}\right)=X_{M} X^{M}=X^{2},
$$

and similarly $\overline{\mathbf{X}} \mathbf{X}=X^{2}$. One also has

$$
\mathbf{X}_{1} \overline{\mathbf{X}}_{2}+\mathbf{X}_{2} \overline{\mathbf{X}}_{1}=\overline{\mathbf{X}}_{1} \mathbf{X}_{2}+\overline{\mathbf{X}}_{2} \mathbf{X}_{1}=2 X_{1} \cdot X_{2}
$$

In the basis defined by eq. (A.5), we have

$$
\left\{\begin{array} { l } 
{ \mathbf { X } _ { \alpha \gamma } = - X ^ { + } \epsilon _ { \alpha \gamma } } \\
{ \mathbf { X } _ { \alpha } ^ { \dot { \gamma } } = - X _ { \mu } \sigma _ { \alpha \dot { \beta } } ^ { \mu } \dot { \beta } ^ { \dot { \beta } \dot { \gamma } } } \\
{ \mathbf { X } _ { \gamma } ^ { \dot { \alpha } } = X _ { \mu } \overline { \sigma } ^ { \mu \dot { \alpha } \beta } \epsilon _ { \beta \gamma } } \\
{ \mathbf { X } ^ { \dot { \alpha } \dot { \gamma } } = X ^ { - } \epsilon ^ { \dot { \alpha } \dot { \gamma } } }
\end{array} \quad \left\{\begin{array}{l}
\overline{\mathbf{X}}^{\alpha \gamma}=-X^{-} \epsilon^{\alpha \gamma} \\
\overline{\mathbf{X}}_{\dot{\gamma}}^{\alpha}=-X_{\mu} \epsilon^{\alpha \beta} \sigma_{\beta \dot{\gamma}}^{\mu} \\
\overline{\mathbf{X}}_{\dot{\alpha}}^{\gamma}=X_{\mu} \epsilon_{\dot{\alpha} \dot{\beta}} \bar{\sigma}^{\mu \dot{\beta} \gamma} \\
\overline{\mathbf{X}}_{\dot{\alpha} \dot{\gamma}}=X^{+} \epsilon_{\dot{\alpha} \dot{\gamma}}
\end{array}\right.\right.
$$


The $4 \mathrm{D}$ spinors are embedded as follows in the 6D chiral spinors (twistors):

$$
\Psi_{a}=\left(\begin{array}{c}
\psi_{\alpha} \\
\bar{\chi}^{\dot{\alpha}}
\end{array}\right), \quad \bar{\Phi}^{a}=\left(\begin{array}{c}
\phi^{\alpha} \\
\bar{\xi}_{\dot{\alpha}}
\end{array}\right) .
$$

In order to avoid a proliferation of spinor indices, we define

$$
\left(\sigma^{\mu} \epsilon\right)_{\alpha}^{\dot{\gamma}} \equiv \sigma_{\alpha \dot{\beta}}^{\mu} \epsilon^{\dot{\beta} \dot{\gamma}}
$$

Notice that in writing eq. (A.16) we have used the usual convention of matrix multiplication. A similar comment applies for other similar expressions involving $\bar{\sigma}^{\mu}, \sigma^{\mu \nu}$ and $\bar{\sigma}^{\mu \nu}$.

\section{B Spinor and vector notation for tensor fields}

We usually write bosonic fields transforming in the lowest representations of the Lorentz group in vector notation: $A_{\mu}, T_{\mu \nu}$, etc. With the notable exception of symmetric traceless tensors of the form $T_{\left(\mu_{1} \ldots \mu_{l}\right)}$, the vector notation becomes awkward for higher spin. On the contrary, by using the isomorphism between $\mathrm{SO}(3,1)$ and $\mathrm{SL}(2, C)$, a generic irreducible representation of the Lorentz group is defined by two integers $(l, \bar{l})$. The matrix $\sigma^{\mu}$ provides the link between the vector and spinor representations of fields. Given a reducible bosonic tensor field $t_{\mu_{1} \ldots \mu_{n}}$ or fermionic spinor-tensor fields $\psi_{\alpha, \mu_{1} \ldots \mu_{n}}, \bar{\psi}_{\mu_{1} \ldots \mu_{n}}^{\dot{\alpha}}$, we have

$$
\begin{aligned}
\left(\sigma^{\mu_{1}} \epsilon\right)_{\alpha_{1}}^{\dot{\beta}_{1}} \ldots\left(\sigma^{\mu_{n}} \epsilon\right)_{\alpha_{n}}^{\dot{\beta}_{n}} t_{\mu_{1} \ldots \mu_{n}} & =\sum_{l, \bar{l}}^{n} t_{\alpha_{1} \ldots \alpha_{l}}^{\dot{\beta}_{1} \ldots \beta_{\bar{l}}} \epsilon_{\alpha_{l+1} \alpha_{l+2}} \ldots \epsilon_{\alpha_{n-1} \alpha_{n}} \epsilon^{\dot{\beta}_{\bar{l}+1} \dot{\beta}_{\bar{l}+2} \ldots \epsilon^{\dot{\beta}_{n-1} \dot{\beta}_{n}}} \\
\left(\sigma^{\mu_{1}} \epsilon\right)_{\alpha_{1}}^{\dot{\beta}_{1}} \ldots\left(\sigma^{\mu_{n}} \epsilon\right)_{\alpha_{n}}^{\dot{\beta}_{n}} \psi_{\gamma \mu_{1} \ldots \mu_{n}} & =\sum_{l, \bar{l}}^{n} \psi_{\gamma \alpha_{1} \ldots \alpha_{l}}^{\dot{\beta}_{1} \ldots \beta_{\bar{l}}} \epsilon_{\alpha_{l+1} \alpha_{l+2}} \ldots \epsilon_{\alpha_{n-1} \alpha_{n}} \epsilon^{\dot{\beta}_{\bar{l}+1} \dot{\beta}_{\bar{l}+2} \ldots \epsilon^{\dot{\beta}_{n-1} \dot{\beta}_{n}}} \\
\left(\sigma^{\mu_{1}} \epsilon\right)_{\alpha_{1}}^{\dot{\beta}_{1}} \ldots\left(\sigma^{\mu_{n}} \epsilon\right)_{\alpha_{n}}^{\dot{\beta}_{n}} \bar{\psi}_{\mu_{1} \ldots \mu_{n}}^{\dot{\gamma}} & =\sum_{l, \bar{l}}^{n} \bar{\psi}_{\alpha_{1} \ldots \alpha_{l}}^{\dot{\gamma} \dot{\beta}_{1} \ldots \beta_{\bar{l}}} \epsilon_{\alpha_{l+1} \alpha_{l+2}} \ldots \epsilon_{\alpha_{n-1} \alpha_{n}} \epsilon^{\dot{\beta}_{\bar{l}+1}} \dot{\beta}_{\bar{l}+2} \ldots \epsilon^{\dot{\beta}_{n-1} \dot{\beta} n}
\end{aligned}
$$

where the sum over $l, \bar{l}$ runs over even or odd integers, for even or odd $n$, respectively. Taking symmetric and antisymmetric combinations in the undotted and dotted indices of the r.h.s. of eq. (B.1) allows us to find the explicit relations between the different field components in vector and spinor notations. Inverse relations are obtained by multiplying eq. (B.1) by powers of $\left(\epsilon \sigma^{\mu}\right)$ :

$$
\begin{aligned}
t_{\mu_{1} \ldots \mu_{n}} & =2^{-n} \sum_{l, \bar{l}}^{n}\left(\epsilon \sigma_{\mu_{1}}\right)_{\dot{\beta}_{1}}^{\alpha_{1}} \ldots\left(\epsilon \sigma_{\mu_{n}}\right)_{\dot{\beta}_{n}}^{\alpha_{n}} t_{\alpha_{1} \ldots \alpha_{l}}^{\dot{\beta}_{1} \ldots \dot{\beta}_{\bar{l}}} \epsilon_{\alpha_{l+1} \alpha_{l+2}} \ldots \epsilon_{\alpha_{n-1} \alpha_{n}} \epsilon^{\dot{\beta}_{\bar{l}+1}} \dot{\beta}_{\bar{l}+2} \ldots \epsilon^{\dot{\beta}_{n-1} \dot{\beta}_{n}} \\
\psi_{\gamma \mu_{1} \ldots \mu_{n}} & =2^{-n} \sum_{l, \bar{l}}^{n}\left(\epsilon \sigma_{\mu_{1}}\right)_{\dot{\beta}_{1}}^{\alpha_{1}} \ldots\left(\epsilon \sigma_{\mu_{n}}\right)_{\dot{\beta}_{n}}^{\alpha_{n}} \psi_{\gamma \alpha_{1} \ldots \alpha_{l}} \dot{\beta}_{\alpha_{l+1} \alpha_{l+2}}^{\dot{\beta}_{1} \dot{\beta}_{\bar{l}}} \ldots \epsilon_{\alpha_{n-1} \alpha_{n}} \epsilon^{\dot{\beta}_{\bar{l}+1} \dot{\beta}_{\bar{l}+2}} \ldots \epsilon^{\dot{\beta}_{n-1} \dot{\beta}_{n}} \\
\bar{\psi}_{\mu_{1} \ldots \mu_{n}}^{\dot{\gamma}} & =2^{-n} \sum_{l, \bar{l}}^{n}\left(\epsilon \sigma_{\mu_{1}}\right)_{\dot{\beta}_{1}}^{\alpha_{1}} \ldots\left(\epsilon \sigma_{\mu_{n}}\right)_{\dot{\beta}_{n}}^{\alpha_{n}} \bar{\psi}_{\alpha_{1} \ldots \alpha_{l}}^{\dot{\gamma}_{\beta_{1}} \dot{\beta}_{1} \dot{\beta}_{\bar{l}}} \epsilon_{\alpha_{l+1} \alpha_{l+2}} \ldots \epsilon_{\alpha_{n-1} \alpha_{n}} \epsilon^{\dot{\beta}_{\bar{l}+1} \dot{\beta}_{\bar{l}+2} \ldots \epsilon^{\dot{\beta}_{n-1} \dot{\beta}_{n}}}
\end{aligned}
$$


It may be useful to work out in detail the case for, say, a bosonic rank-two tensor $t_{\mu \nu}$. We have

$$
\left(\sigma^{\mu} \epsilon\right)_{\alpha_{1}}^{\dot{\beta}_{1}}\left(\sigma^{\nu} \epsilon\right)_{\alpha_{2}}^{\dot{\beta}_{2}} t_{\mu \nu}=t \epsilon_{\alpha_{1} \alpha_{2}} \epsilon^{\dot{\beta}_{1} \dot{\beta}_{2}}+t_{\alpha_{1} \alpha_{2}} \epsilon^{\dot{\beta}_{1} \dot{\beta}_{2}}+t^{\dot{\beta}_{1} \dot{\beta}_{2}} \epsilon_{\alpha_{1} \alpha_{2}}+t_{\alpha_{1} \alpha_{2}}^{\dot{\beta}_{1} \dot{\beta}_{2}}
$$

which corresponds to the decomposition $(0,0) \oplus(1,0) \oplus(0,1) \oplus(1,1)$, scalar, self-dual antisymmetric tensor, anti self-dual antisymmetric tensor, symmetric tensor. From eq. (B.3) we get

$$
\begin{aligned}
t & =\frac{1}{2} \eta^{\mu \nu} t_{\mu \nu}, \\
t_{\alpha_{1} \alpha_{2}} & =t_{\mu \nu}\left(\sigma^{\mu \nu} \epsilon\right)_{\alpha_{1} \alpha_{2}}, \\
t^{\dot{\beta}_{1} \dot{\beta}_{2}} & =t_{\mu \nu}\left(\epsilon \bar{\sigma}^{\mu \nu}\right)^{\dot{\beta}_{1} \dot{\beta}_{2}} \\
t_{\alpha_{1} \alpha_{2}}^{\dot{\beta}_{1} \dot{\beta}_{2}} & =\frac{1}{4} t_{\mu \nu}\left(\left(\sigma^{\mu} \epsilon\right)_{\alpha_{1}}^{\dot{\beta}_{1}}\left(\sigma^{\nu} \epsilon\right)_{\alpha_{2}}^{\dot{\beta}_{2}}+\left(\sigma^{\mu} \epsilon\right)_{\alpha_{2}}^{\dot{\beta}_{1}}\left(\sigma^{\nu} \epsilon\right)_{\alpha_{1}}^{\dot{\beta}_{2}}+(\mu \leftrightarrow \nu)\right) .
\end{aligned}
$$

Notice that in the last relation in eq. (B.4) the trace part of $t_{\mu \nu}$ automatically gives a vanishing contribution. We get the inverse relations by means of eq. (B.2). Decomposing $t_{\mu \nu}=$ $\eta_{\mu \nu} t / 2+t_{[\mu \nu]}+t_{(\mu \nu)}$, where $t_{(\mu \nu)}=1 / 2\left(t_{\mu \nu}+t_{\nu \mu}\right)-\eta_{\mu \nu} t / 2$ and $t_{[\mu \nu]}=1 / 2\left(t_{\mu \nu}-t_{\nu \mu}\right)$, one has

$$
\begin{aligned}
t_{[\mu \nu]} & =\frac{1}{2}\left(\epsilon \sigma_{\mu \nu}\right)^{\alpha_{1} \alpha_{2}} t_{\alpha_{1} \alpha_{2}}+\frac{1}{2}\left(\bar{\sigma}_{\mu \nu} \epsilon\right)_{\dot{\beta}_{1} \dot{\beta}_{1}} t^{\dot{\beta}_{1} \dot{\beta}_{2}}, \\
t_{(\mu \nu)} & =\left(\epsilon \sigma_{\mu}\right)_{\dot{\beta}_{1}}^{\alpha_{1}}\left(\epsilon \sigma_{\nu}\right)_{\dot{\beta}_{2}}^{\alpha_{2}} t_{\dot{\alpha}_{1} \alpha_{2}}^{\dot{\beta}_{1} \dot{\beta}_{2}} .
\end{aligned}
$$

For arbitrary symmetric traceless fields $t_{\left(\mu_{1} \ldots \mu_{l}\right)}$, in particular, we have

$$
\begin{aligned}
t_{\alpha_{1} \ldots \alpha_{l}}^{\dot{\beta}_{1} \dot{\beta}_{l}} & =\frac{1}{l !} t_{\left(\mu_{1} \ldots \mu_{l}\right)}\left(\left(\sigma^{\mu_{1}} \epsilon\right)_{\alpha_{1}}^{\dot{\beta}_{1}} \ldots\left(\sigma^{\mu_{l}} \epsilon\right)_{\alpha_{l}}^{\dot{\beta}_{l}}+\text { perms. }\right) \\
t_{\left(\mu_{1} \ldots \mu_{l}\right)} & =\left(\epsilon \sigma_{\mu_{1}}\right)_{\dot{\beta}_{1}}^{\alpha_{1}} \ldots\left(\epsilon \sigma_{\mu_{l}}\right)_{\dot{\beta}_{l}}^{\alpha_{l}} t_{\alpha_{1} \ldots \alpha_{l}}^{\dot{\beta}_{1} \ldots \dot{\beta}_{l}}
\end{aligned}
$$

Open Access. This article is distributed under the terms of the Creative Commons Attribution License (CC-BY 4.0), which permits any use, distribution and reproduction in any medium, provided the original author(s) and source are credited.

\section{References}

[1] H. Osborn and A.C. Petkou, Implications of conformal invariance in field theories for general dimensions, Annals Phys. 231 (1994) 311 [hep-th/9307010] [INSPIRE].

[2] J. Erdmenger and H. Osborn, Conserved currents and the energy momentum tensor in conformally invariant theories for general dimensions, Nucl. Phys. B 483 (1997) 431 [hep-th/9605009] [INSPIRE].

[3] J.M. Maldacena and G.L. Pimentel, On graviton non-Gaussianities during inflation, JHEP 09 (2011) 045 [arXiv: 1104.2846] [INSPIRE].

[4] S. Giombi, S. Prakash and X. Yin, A note on CFT correlators in three dimensions, JHEP 07 (2013) 105 [arXiv: 1104.4317] [INSPIRE].

[5] J. Maldacena and A. Zhiboedov, Constraining conformal field theories with a higher spin symmetry, J. Phys. A 46 (2013) 214011 [arXiv:1112.1016] [INSPIRE]. 
[6] A. Zhiboedov, A note on three-point functions of conserved currents, arXiv:1206.6370 [INSPIRE].

[7] Y.S. Stanev, Correlation functions of conserved currents in four dimensional conformal field theory, Nucl. Phys. B 865 (2012) 200 [arXiv:1206.5639] [INSPIRE].

[8] A. Dymarsky, On the four-point function of the stress-energy tensors in a CFT, arXiv: 1311.4546 [INSPIRE].

[9] M.S. Costa, J. Penedones, D. Poland and S. Rychkov, Spinning conformal correlators, JHEP 11 (2011) 071 [arXiv:1107.3554] [InSPIRE].

[10] S. Weinberg, Six-dimensional methods for four-dimensional conformal field theories, Phys. Rev. D 82 (2010) 045031 [arXiv: 1006.3480] [inSPIRE].

[11] G.M. Sotkov and R.P. Zaikov, Conformal invariant two point and three point functions for fields with arbitrary spin, Rept. Math. Phys. 12 (1977) 375 [INSPIRE].

[12] R. Rattazzi, V.S. Rychkov, E. Tonni and A. Vichi, Bounding scalar operator dimensions in 4D CFT, JHEP 12 (2008) 031 [arXiv:0807.0004] [INSPIRE].

[13] D. Simmons-Duffin, Projectors, shadows and conformal blocks, JHEP 04 (2014) 146 [arXiv: 1204.3894] [INSPIRE].

[14] M.S. Costa and T. Hansen, Conformal correlators of mixed-symmetry tensors, arXiv: 1411.7351 [INSPIRE].

[15] P.A.M. Dirac, Wave equations in conformal space, Annals Math. 37 (1936) 429 [INSPIRE].

[16] S. Ferrara, A.F. Grillo and R. Gatto, Tensor representations of conformal algebra and conformally covariant operator product expansion, Annals Phys. 76 (1973) 161 [INSPIRE].

[17] S. Ferrara, R. Gatto and A.F. Grillo, Conformal algebra in space-time and operator product expansion, Springer Tracts Mod. Phys. 67 (1973) 1 [INSPIRE].

[18] M.S. Costa, J. Penedones, D. Poland and S. Rychkov, Spinning conformal blocks, JHEP 11 (2011) 154 [arXiv:1109.6321] [INSPIRE].

[19] V.K. Dobrev, G. Mack, V.B. Petkova, S.G. Petrova and I.T. Todorov, Harmonic analysis on the $n$-dimensional Lorentz group and its application to conformal quantum field theory, Springer, Berlin Germany (1977).

[20] W. Siegel, Green-Schwarz formulation of selfdual superstring, Phys. Rev. D 47 (1993) 2512 [hep-th/9210008] [INSPIRE].

[21] W. Siegel, Embedding versus 6D twistors, arXiv:1204.5679 [INSPIRE].

[22] W.D. Goldberger, W. Skiba and M. Son, Superembedding methods for $4 d N=1$ SCFTs, Phys. Rev. D 86 (2012) 025019 [arXiv:1112.0325] [INSPIRE].

[23] W.D. Goldberger, Z.U. Khandker, D. Li and W. Skiba, Superembedding methods for current superfields, Phys. Rev. D 88 (2013) 125010 [arXiv:1211.3713] [INSPIRE].

[24] A.L. Fitzpatrick et al., Covariant approaches to superconformal blocks, JHEP 08 (2014) 129 [arXiv: 1402.1167] [INSPIRE].

[25] Z.U. Khandker, D. Li, D. Poland and D. Simmons-Duffin, $N=1$ superconformal blocks for general scalar operators, JHEP 08 (2014) 049 [arXiv:1404.5300] [INSPIRE].

[26] G. Mack, Convergence of operator product expansions on the vacuum in conformal invariant quantum field theory, Commun. Math. Phys. 53 (1977) 155 [INSPIRE]. 
[27] G. Mack, All unitary ray representations of the conformal group $\mathrm{SU}(2,2)$ with positive energy, Commun. Math. Phys. 55 (1977) 1 [INSPIRE].

[28] S. Minwalla, Restrictions imposed by superconformal invariance on quantum field theories, Adv. Theor. Math. Phys. 2 (1998) 781 [hep-th/9712074] [InSPIRE].

[29] V.K. Dobrev, E.K. Khristova, V.B. Petkova and D.B. Stamenov, Conformal covariant operator product expansion (OPE) of two spin 1/2 fields, Bulg. J. Phys. 1 (1974) 42 [INSPIRE].

[30] J. Wess and J. Bagger, Supersymmetry and supergravity, second edition, Princeton University Press, Princeton U.S.A. (1991). 